\title{
Paucity of Pericytes in Germinal Matrix Vasculature of Premature Infants
}

\author{
Alex Braun, ${ }^{1}$ Hongmin $\mathrm{Xu},{ }^{2}$ Furong Hu, ${ }^{2}$ Praneeth Kocherlakota, ${ }^{3}$ Donald Siegel, ${ }^{3}$ Praveen Chander, ${ }^{1}$ Zoltan Ungvari, ${ }^{4}$ \\ Anna Csiszar, ${ }^{4}$ Maiken Nedergaard, ${ }^{5}$ and Praveen Ballabh ${ }^{2,3}$ \\ Departments of ${ }^{1}$ Pathology, ${ }^{2}$ Pediatrics, ${ }^{3}$ Anatomy and Cell Biology, and ${ }^{4}$ Physiology, New York Medical College, Westchester Medical Center, Valhalla, New \\ York 10595, and ${ }^{5}$ Neurosurgery and Center of Aging and Developmental Biology, University of Rochester, Rochester, New York 14642
}

Germinal matrix (GM) is a richly vascularized collection of neuronal-glial precursor cells in the developing brain, which is selectively vulnerable to hemorrhage in premature infants. It has rapid angiogenesis associated with high levels of vascular endothelial growth factor (VEGF). Because pericytes provide structural stability to blood vessels, we asked whether pericytes were fewer in the GM than in the subjacent white matter and neocortex and, if so, whether angiogenic inhibition could increase the pericyte density in the GM. We found pericyte coverage and density less in the GM vasculature than in the cortex or white matter in human fetuses, premature infants, and premature rabbit pups. Notably, although VEGF suppression significantly enhanced pericyte coverage in the GM, it remained less than in other brain regions. Therefore, to further elucidate the basis of fewer pericytes in the GM vasculature, we examined expression of ligand-receptor systems responsible for pericyte recruitment. Transforming growth factor- $\beta 1$ (TGF- $\beta 1$ ) protein expression was lower, whereas sphingosine-1-phosphate1 (S1P1) and N-cadherin levels were higher in the GM than in the cortex or white matter. Low TGF- $\beta 1$ may be involved in promoting endothelial proliferation, whereas elevated S1P1 with $\mathrm{N}$-cadherin may assist vascular maturation. Hence, a paucity of pericytes in the GM vasculature may contribute to its propensity to hemorrhage, and a lower expression of TGF- $\beta 1$ could be a basis of reduced pericyte density in its vasculature.

Key words: GM; intraventricular hemorrhage; GM hemorrhage; pericytes; cortex; white matter; PDGF-B; PDGFR- $\beta$; TGF- $\beta$; ALK-1; ALK-5; N-cadherin; sphingosine 1 phosphate

\section{Introduction}

In the United States, $\sim 12,240$ premature infants develop germinal matrix hemorrhage-intraventricular hemorrhage (GMHIVH) every year (Investigators of the Vermont-Oxford Trial Network Database Project, 1993; Heuchan et al., 2002). A large number of these infants develop cerebral palsy, hydrocephalus, and mental retardation (du Plessis and Volpe, 2002; Ballabh et al., 2004a). The germinal matrix (GM), located in the thalamostriate groove beneath the forebrain ependyma, is a richly vascularized collection of neural precursor cells that persists until late gestation.

The GM has greater vascular density than either the adjacent white matter or neocortical mantle (Ballabh et al., 2004b), suggesting a greater metabolic rate and oxygen requirement compared with other brain regions. More importantly, we reported that a rapid angiogenesis in the GM, induced by high vascular endothelial growth factor (VEGF) and angiopoietin (ANGPT)-2 levels, is associated with greater vascular fragility and propensity

Received July 19, 2007; revised Sept. 9, 2007; accepted Sept. 14, 2007.

This work was supported by National Institutes of Health-National Institute of Neurological Disorders and Stroke Grant NS050586 (P.B.). We thank Dr. J. Etlinger for support and critical evaluation of this manuscript and J. Abrahams and Yiping Xing for their technical assistance.

Correspondence should be addressed to Dr. Praveen Ballabh, Regional Neonatal Center, Maria Fareri Children's Hospital at Westchester Medical Center, Valhalla, NY 10595. E-mail: pballabh@msn.com.

D0I:10.1523/JNEUROSCI.3281-07.2007

Copyright $\odot 2007$ Society for Neuroscience ～0270-6474/07/2712012-13\$15.00/0 to hemorrhage, and that angiogenic inhibition reduces both the incidence and the severity of GMH in rabbit pup model (Ballabh et al., 2007). Angiogenesis involves formation of nascent blood vessels, which are subsequently stabilized by pericytes. Pericytes are perivascular cells with multifunctional activities (Balabanov and Dore-Duffy, 1998). They are involved in various stages of angiogenesis including initiation, sprout extension, migration, and maturation of blood vessels (Hirschi and D'Amore, 1997). In response to angiogenic stimuli, they degrade basal lamina to migrate out of the blood vessels, and also regulate endothelial proliferation and migration (Jain, 2003). At the end of angiogenesis, they resume their position and strengthen vessels by synthesis of extracellular matrix and inducing endothelial differentiation (Armulik et al., 2005). Intriguingly, abnormal and depressed angiogenesis is found in both mice treated with antibodies targeted against pericytes and pathological phenotypes of mice in which pericyte development is blocked (Lindahl et al., 1997; Hellstrom et al., 2001; Ozerdem, 2006). There are at least four ligand-receptor systems involved in pericyte recruitment and vessel maturation (Jain, 2003). These include TGF- $\beta 1$ and its receptors, platelet-derived growth factor-B (PDGF-B) and its receptor (PDGFR- $\beta$ ), ANGPT and its Tie receptor, and sphingosine 1 phosphate (S1P) and its receptor S1P1 (also known as EDG-1). Mouse embryos lacking any of these ligands or their receptors develop primary vasculature, but endothelial cells fail to associate with pericytes resulting in angiogenic defects with an enhanced 
propensity to hemorrhage (Lindahl et al., 1997; Yancopoulos et al., 2000; Jain, 2003). Indeed, pericytes provide stability and structural integrity to the microvasculature. On this basis, we hypothesized that GM vasculature might have a lower density of pericytes compared with other brain regions, and that angiogenic inhibition with VEGF inhibitors might increase pericyte density. Because ANGPT, PDGF-B, TGF- $\beta 1$, and S1P, as well as their receptors, are necessary for pericyte recruitment around the endothelial tube, we also postulated that there might be differential expression of these growth factors and their receptors in the GM compared with the cortex or white matter leading to reduced pericyte recruitment, which in turn could enhance the vulnerability of the GM microvasculature to hemorrhage.

This study demonstrated that the pericyte coverage and density were lower in the GM vasculature compared with the other brain regions in human fetuses, premature infants, and premature rabbit pups. In addition, angiogenic inhibition with celecoxib (COX-2- and VEGF inhibitor) or N-(4-bromo-2-fluorophenyl)-6methoxy-7-[(1-methylpiperidin-4-yl)methoxy] quinazolin-4-amine (ZD6474) (VEGFR2 inhibitor) increased pericyte coverage and density in the GM vasculature of rabbit pups. Importantly, TGF- $\beta 1$ protein expression was lower, whereas $\mathrm{S} 1 \mathrm{P} 1$ and $\mathrm{N}$-cadherin levels were higher in the human GM than the cortex or white matter.

\section{Materials and Methods}

Human subjects. The Institutional Review Board at New York Medical College and Westchester Medical Center (Valhalla, NY) approved the use of autopsy materials from fetuses and premature infants for this study. The study material included brain samples from premature infants of postconceptional age 23-40 weeks and from spontaneous abortuses of the gestational age 16-22 weeks. Gestational age was based on the last menstrual period of the mother. Only autopsy samples of $<18$ h postmortem interval for premature infants and $<8 \mathrm{~h}$ for fetuses were used for this study. All infants with major congenital anomalies, chromosomal defects, culture-proven sepsis, meningitis, and hypoxic-ischemic encephalopathy, and infants receiving extracorporeal membrane oxygenator treatment were excluded from the study. In addition, brains with autolytic changes, clearly observable gross brain pathologies, or those seen by microscopy of hematoxylin and eosin-stained sections were excluded. Gestational age, sex, weight, Apgar score, relevant clinical problems, cause of death, and prenatal as well as postnatal medications were recorded on datasheets. This information was obtained from the pathologist in such a manner that subjects could not be identified directly or through identifiers linked to subjects. We included 9 human fetuses (16-22 weeks), 12 premature (23-35 weeks), and 3 mature (36-40 weeks) infants for Western blot analysis and real-time PCR for all markers. To quantify pericytes by immunolabeling, we used materials from 5 human fetuses, 10 premature and 3 full-term infants; and for electron microscopy, we used 12 subjects of 20-28 week gestational age. The terminated fetuses (16-22 weeks; $n=8)$ appeared healthy, but were nonviable. Among early premature infants (23-27 weeks; $n=7$ ), five infants died of respiratory distress syndrome (premature lung) and prematurity, one died of culture-negative clinical sepsis, and one died of prematurity with persistent pulmonary hypertension. Among late premature infants ( $28-37$ weeks; $n=6$ ), the cause of death was necrotizing enterocolities (one infant), perforated ileum (one infant), severe pulmonary atresia (one infant), respiratory failure (one infant), hypoplastic lung (one infant), and culture negative clinical sepsis (one infant). Of three mature infants, two were stillborn and one had cardiomyopathy.

Human tissue collection and processing. The wall of fetal cerebral hemisphere consists of ventricular zone, subventricular zone, intermediate zone, cortical plate, and marginal zone as described by Angevine et al. (1970). In the present study, for the sake of uniformity and consistency of presentation, white matter refers to intermediate zone embryonic white matter, and cortex refers to neocortical mantle. Brain samples were processed as described previously (Ballabh et al., 2007). Briefly, we obtained
3- to 4-mm-thick coronal blocks by sectioning through frontal cortex, frontal white matter, and GM in the region of thalamostriate groove and at the level of foramen of Monro. The samples were fixed in $4 \%$ paraformaldehyde in PBS (0.01 M PBS, pH 7.4) for $\sim 18 \mathrm{~h}$, and then were cryoprotected by immersing into $20 \%$ sucrose in $0.01 \mathrm{M}$ PBS buffer for $24 \mathrm{~h}$ followed by $30 \%$ sucrose for the next $24 \mathrm{~h}$. Tissues were frozen after embedding them into OCT compound (Sakura, Tokyo, Japan). Frozen coronal blocks were cut into $12 \mu \mathrm{m}$ sections using cryostat and saved at $-80^{\circ} \mathrm{C}$ until use. To identify GM, sections of tissues taken from periventricular area of the thalamostriate groove were stained with hematoxylin and eosin, and were examined under a light microscope.

Immunohistochemistry. The primary antibodies used in the experiments included polyclonal rabbit PDGF-B (catalog \#SC7878; Santa Cruz Biotechnology, Santa Cruz, CA), polyclonal rabbit PDGFR- $\beta$ (catalog \#SC339; Santa Cruz Biotechnology), polyclonal goat TGF- $\beta 1$ (catalog \#SC146; Santa Cruz Biotechnology), polyclonal rabbit activin receptorlike kinase 1 (ALK-1) (catalog \#SC19546; Santa Cruz Biotechnology), polyclonal rabbit ALK-5 (catalog \#SC9048; Santa Cruz Biotechnology), monoclonal $\alpha$-smooth muscle actin (catalog \#A2547; Sigma, St. Louis, MO), rabbit polyclonal sphingosine 1 phosphate 1 (catalog \#10005228; Cayman Chemicals, Ann Arbor, MI), goat polyclonal PECAM-1 (catalog SC1506; Santa Cruz Biotechnology), CD34 QBEnd/10 (catalog \#326-01; Signet, Dedham, MA), mouse anti human chondroitin sulfate proteoglycan (NG2) monoclonal antibody (Chemicon MAB; catalog \#2029; Chemicon, Temecula, CA), mouse monoclonal N-Cadherin (catalog \#33-3900; Zymed, San Francisco, CA), and polyclonal desmin (catalog \#D8281; Sigma).

Secondary antibodies (all from Jackson ImmunoResearch, West Grove, PA) were Cy-5 conjugate goat-anti-mouse (catalog \#115-175146), rhodamine conjugate goat anti-rabbit (catalog \#111-295-144), Cy-5 conjugate goat anti-rabbit (catalog \#111-175-144), Cy3 conjugate donkey anti-goat (catalog \#705-165-147), Cy5 conjugate donkey antimouse (catalog \#715-175-150), Cy3 conjugate IgG1-specific goat antimouse antibody (catalog \#115-175-205), and Cy 5 conjugate IgG2aspecific goat anti-mouse (catalog \#115-165-206) antibodies. Immunohistochemistry was performed as described previously (Ballabh et al., 2005). Briefly, tissue sections were incubated overnight at $4^{\circ} \mathrm{C}$ with the primary antibody diluted in PBS. After several washes in PBS, sections were incubated in secondary antibody for $1 \mathrm{~h}$ at room temperature. Finally, after washes in PBS, sections were mounted with Slow Fade Light Antifade reagent (Invitrogen, Carlsbad, CA) and were visualized under a Bio-Rad (MRC-1024ES; Bio-Rad, Hercules, CA) confocal microscope. Controls were prepared by replacing primary antibodies with $0.2 \%$ Triton X-100 in PBS.

Double and triple labeling. To visualize pericytes in relation to the endothelium in the rabbit pup brain, we double labeled brain sections with a combination of a mouse monoclonal NG2 and goat polyclonal PECAM-1 antibody. To demonstrate pericytes in human materials, we performed triple labeling of the brain sections from fetuses and premature infants using mouse monoclonal NG2 (IgG2a), rabbit polyclonal PDGFR- $\beta$, and mouse monoclonal CD34 antibodies (IgG1). We could not obtain satisfactory immunostaining with the commercially available PDGFR- $\beta$ antibody in rabbit brain samples, and thus immunohistochemistry for PDGFR- $\beta$ in rabbit tissues was precluded from the study. Immunolabeling with primary antibodies was done sequentially for double and triple labeling, as described previously (Ballabh et al., 2007).

Quantifications. Images were analyzed using MetaMorph version 6.1 from Universal Imaging Corporation (West Chester, PA) 1993-2003 as described previously (El-Khoury et al., 2006). From each brain, a set of three to five coronal sections, taken as every 10th section, was used for the study. From every section, approximately five images were acquired from each area (GM, cortex, and white mater) using a $60 \times$ objective. Thus, a total of 45-75 images (3-5 sections $\times 3$ areas $\times 5$ images) per subject were acquired, and a total of 1177 blood vessel profiles were analyzed from 18 human subjects [5 fetuses (16-22 weeks), 5 early premature infants (23-27 weeks), 5 late premature infants ( $28-35$ weeks), and 3 mature infants (36-40 weeks)]. Of 1177 blood vessel profiles, 349 were from cortex, 358 were from white matter, and 470 were from the GM.

We determined pericyte coverage of endothelial cells and pericyte den- 
sity. NG2 and PDGFR- $\beta$ antibodies were used to label pericytes, and CD34-specific antibody to label endothelia. Two-dimensional images were acquired and analyzed for the overlapping of the perimeter of blood vessels and pericytes. For triple labeling, endothelium stained with CD34-specific antibody was seen in red (Cy3 florescent probe), pericyte stained with anti-NG2 seen as green (FITC), and pericyte labeled with PDGFR- $\beta$ as blue (Cy5). MetaMorph, version 6.1, software (Universal Imaging Corporation) was used for analysis. The two sources of image, endothelium (red) and pericyte (blue or green), were displayed on the MetaMorph screen. Both sources of images were thresholded. "Region tool" in the software was then used to define and select the outer margin of the blood vessel under consideration. The software then measured this delineated area in pixels and calculated the percentage overlap (colocalization) of red (endothelium) over blue/green signal (pericyte). Thus, each blood vessel (endothelia) margin in every image was individually selected to evaluate overlap with the pericyte. Pericyte density was quantified by calculating the ratio of NG2/PDGFR- $\beta$-stained area and CD34stained area in human samples. We also measured diameter (caliper width of the object perpendicular to the largest chord) of blood vessels.

From each premature rabbit pup brain [embryonic day 29 (E29)], a set of two to three coronal sections $(12 \mu \mathrm{m})$ taken as every 10th section was used for the study. From every section, approximately four images were acquired from each area (GM, cortex, and white mater) using a $60 \times$ objective. A total of 165 blood vessels profiles ( $n=5$ pups) were evaluated among untreated control pups. Of 165 blood vessel profiles, 52, 50, and 63 blood vessels were from the cortex, white matter, and GM, respectively. In addition to controls, we also evaluated similar number of brain sections and blood vessel profiles from pups exposed to prenatal celecoxib ( $n=5$ pups) and ZD6474 ( $n=5$ pups). In rabbits, we only used NG2 antibodies to evaluate pericytes because we could not achieve satisfactory immunostaining with PDGFR- $\beta$. We calculated pericyte density and coverage in the same manner as described above for human subjects. In addition, we measured the vessel density (number of blood vessels per square millimeter) and percent blood vessel area (percentage of the area occupied by cross section of all blood vessels in an image) in control rabbit pups compared with ZD6474- or celecoxib-treated pups. We used PECAM-1-specific antibody to evaluate blood vessels in rabbits.

Western blot analyses. Protein extraction and Western blotting were performed under reducing conditions as described previously (Ballabh et al., 2005). Brain samples of $\sim 200 \mathrm{mg}$ weight were taken from white matter, cerebral cortex, and GM, placed in a Microfuge tube, frozen on dry ice, and stored at $-80^{\circ} \mathrm{C}$ until use. Frozen brain tissue was homogenized in $500 \mu \mathrm{l}$ of sample buffer (3\% SDS, 10\% glycerol, $62.5 \mathrm{~mm}$ Tris$\mathrm{HCl}$, and $100 \mathrm{~mm}$ DTT) using a mechanical homogenizer, and boiled immediately for $5 \mathrm{~min}$. The protein concentration in the sample was determined using RC DC protein assay kit (Bio-Rad) and dilutions of BSA used as the standard. Total protein samples were separated by SDSPAGE. Equal amounts of protein $(30 \mu \mathrm{g})$ were loaded onto $4-15 \%$ gradient precast gel (Bio-Rad). The separated proteins were transferred to polyvinylidene difluoride membrane by electrotransfer. The membranes were then incubated with primary antibodies, washed, incubated with secondary antibody, and washed again. Target proteins were detected with chemiluminescence ECL system (Amersham Biosciences, Piscataway, NJ) by using secondary antibodies conjugated with horseradish peroxidase (Jackson ImmunoResearch). The blots were then stripped with stripping buffer (Pierce, Rockford, IL) and incubated with $\beta$-actin primary antibody (to detect loading inequalities) followed by secondary antibody and detection with chemiluminescence ECL system. Blots from each experiment were densitometrically analyzed and values were normalized by taking ratio of PDGF, PDGFR- $\beta 1$, TGF- $\beta$, ALK-5, SIP1, and $\mathrm{N}$-cadherin to $\beta$-actin. Satisfactory immunoblot with commercially available ALK-1 antibodies (Santa Cruz Biotechnology) could not be achieved.

Quantitative real-time PCR on human samples. RNA was extracted from human brain tissues using RNAeasy Lipid Tissue Mini kit (catalog \#74804; Qiagen, Hilden, Germany) according to the manufacturer's protocol. Total RNA was reverse transcripted to cDNAs by using SuperScript First-Strand Synthesis System for RT-PCR kit (catalog \#11904-018; Invitrogen) following the manufacturer's protocol. Real-time PCR was used to quantify mRNA expression using the Stratagene (Bernried, Germany) MX3000 on the cDNA as described (Ballabh et al., 2007). Samples were run in triplicate. Efficiency of the PCR was determined using a dilution series of brain mRNA samples. Quantification was performed using the $\Delta \Delta$ Ct method. The housekeeping gene, GAPDH, was used for internal normalization. Fidelity of the PCR was determined by melting temperature analysis and visualization of product on a $2 \%$ agarose gel.

Oligonucleotides used for quantitative real-time-PCR are as follows: human PDGF-B (accession number NM_002608) TGTTGAGGTGGTGTAGATGG (sense) and GGTGGAGCAGACTGAAGG (antisense); PDGFR- $\beta$ (accession number NM_002609) TTAATGCTGGAGGCTGAG (sense) and TGGATGGATTAAGACTGAGG (antisense). TaqMan probes for TGF- $\beta 1$ (catalog \#Hs99999918_m1; NM_000660.3), ALK-1 (catalog \#Hs00163543_m1; NM_000020.1), ALK-5 (catalog\#Hs00610318_m1; NM_004612.2), S1P1 (catalog \#Hs00173499_m1; NM_001400.3), N-cadherin (catalog \#HS00169953_ml; NM_001792.2), and GAPDH (catalog \#HS99999905_m1; NM_002046.3) were bought from Applied Biosystems (Foster City, CA) to run real-time PCR.

Electron microscopy. For electron microscopy, 1-2 mm cubes of tissues from human periventricular GM, cerebral cortex, and white matter were immersion fixed into $2.5 \%$ gluteraldehyde overnight. The tissues were washed in $0.1 \mathrm{~m}$ sodium cacodylate buffer, $\mathrm{pH} 7.4$, postfixed in buffered osmium tetroxide for 1-2 h, stained en bloc with $1 \%$ uranyl acetate, dehydrated in graded ethanol solutions, and then embedded in epoxy resin. The thick sections stained with cresyl violet were seen under light microscope to identify the brain region. Sections of 60-90 nm thicknesses were mounted onto grids and were examined under Leo 906 electron microscope. We processed a total of 12 postmortem human brain samples from fetuses and premature infants of 20-28 week gestational age. In addition, we included 12 rabbit (E29) pup brains (from four different litters) in the study. To obtain rabbit brain samples from three brain regions (cortex, white matter, and GM), we cut 2-mm-thick slices of freshly harvested rabbit pup brain (E29) using Brain Matrix (Precision Instruments, Sarasota, FL). We then dissected the second and third brain slice after floating them in PBS in a silicone (Slygard; Precision Instruments)-coated Petri dish under SteReo discovery microscope (Carl Zeiss, Oberkochen, Germany). The dissected cortex, white matter, and GM were processed similarly to human tissues. Electron micrographs were evaluated by two pathologists separately who were blinded to the origin of the images (cortex, white matter, or GM).

\section{Animal experiments}

Design. Animal protocol was approved by Institutional Animal Care and Use Committee of New York Medical College (Valhalla, NY). We obtained nine timed pregnant New Zealand rabbits from Charles River Laboratories (Wilmington, MA). The rabbits were sequentially assigned to receive celecoxib, ZD6474, or no treatment. C-section was performed at day 29 of gestational age. Pups were dried immediately and their mouths were suctioned. They were kept warm in an infant incubator at $35^{\circ} \mathrm{C}$ and $80 \%$ humidity. After stabilization of their conditions, we weighed the pups. Pups were killed within $2 \mathrm{~h}$ of $\mathrm{C}$-section after sedating with isoflurane. Brain was dissected and cut into $2 \mathrm{~mm}$ coronal slices on brain matrix. Brain slices were fixed in $4 \%$ paraformaldehyde and were processed similar to human tissues.

Celecoxib treatment. Pregnant rabbits received celecoxib (celebrex; Pfizer, Groton, CT) in a dose of $20 \mathrm{mg} \cdot \mathrm{kg}^{-1} \cdot$ dose $^{-1}$ twice daily on 26, 27, and $28 \mathrm{~d}$ of gestational age. Celecoxib was administered orally by mixing content of the capsule in water. We used a feeding catheter to deliver celecoxib into the oral cavity of the dam.

ZD6474 treatment. Pregnant rabbits were treated with oral ZD6474 (AstraZeneca, Wilmington, DE) once daily $(20 \mathrm{mg} / \mathrm{kg}$ ) on 26,27 , and 28 d gestational age. We administered it to pregnant rabbits just as oral celecoxib.

Untreated controls as well as pups treated with prenatal cele- 
CD34 NG2 PDGIR- $[$ P
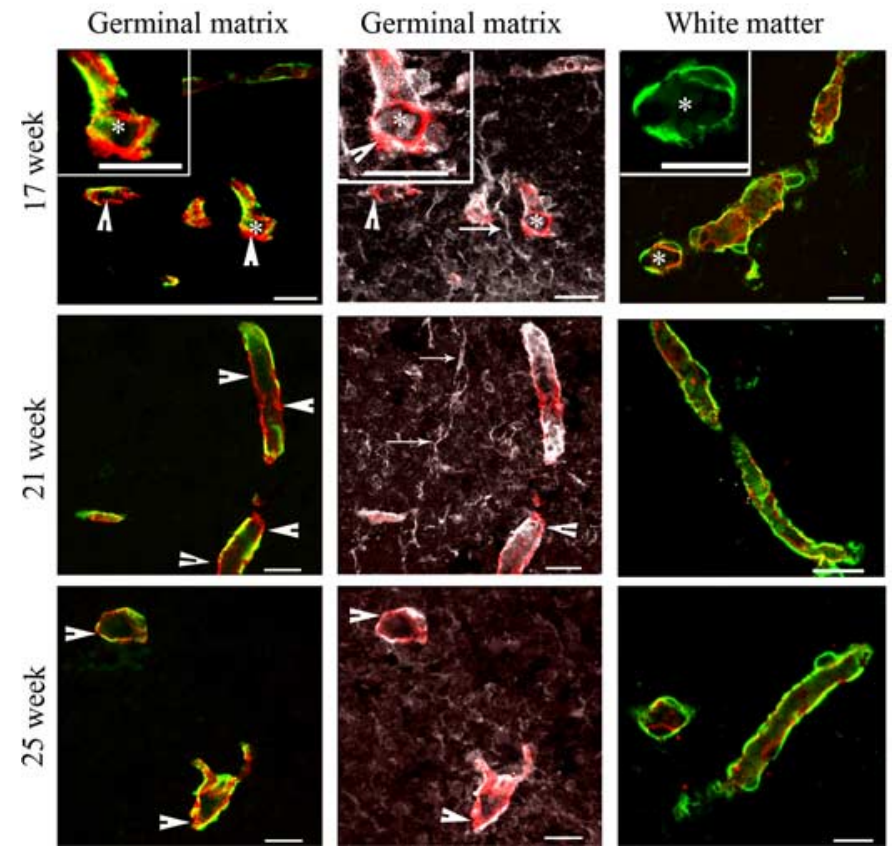

Figure 1. Lesser NG2- and PDGFR- $\beta$-positive pericyte coverage in human GM vasculature. Representative immunofluorescence of cryosections from the GM and white matter (WM) of a 17 and 21 week fetus as well as a 25 week premature infant. Sections were triple labeled with CD34 (endothelial marker; red)-, NG2 (pericyte marker; green)-, and PDGFR- $\beta$ (pericyte marker; white)-specific antibodies. In both GM and white matter, NG2- and PDGFR- $\beta$-positive pericytes were intimately associated with the endothelial surface. The coverage of the endothelia by NG2- and PDGFR- $\beta$-positive pericytes was interrupted (arrowhead) and incomplete in the GM. In contrast, in the white matter, the vascular coverage by pericytes was relatively complete. The insets show typical GM blood vessel and white matter vessel under higher magnification. The asterisk indicates vessel shown under high magnification. GM vessel (merge image) has less pericyte coverage than white matter vessel (pericyte image). PDGFR- $\beta$ immunoreactivity was also observed on radial glia (arrow) in the GM. Scale bar, $20 \mu \mathrm{m}$.

coxib or ZD6474 were euthanized at $2 \mathrm{~h}$ of age, and their brains were processed for immunohistochemistry.

\section{Statistics and analysis}

We analyzed pericyte density and pericyte coverage as well as the expression of growth factors and their receptors, PDGF-B, PDGFR- $\beta$, TGF- $\beta$, ALK-1, ALK-5, S1P1, and N-cadherin, as a function of gestational age. To assess the effect of gestational age on these molecules, human subjects were stratified in four gestational age categories: 16-22 weeks (fetuses), 23-27 weeks (younger prematures), 28-35 weeks (older prematures), and 36-40 weeks (matures). The mean values of protein and mRNA level were presented as bar diagram to assess whether the data followed an apparent trend with the advancing gestational age. We compared mean values of protein and mRNA in the cortex, white matter, and GM. To assess the effect of celecoxib and ZD6474 on pericyte density, pericyte coverage, vascular density, and percent vessel area, we compared these metrics between pups treated with these agents and untreated controls. We used Mann-Whitney $U$ test to perform pairwise comparison and ANOVA to compare more than two groups. A value of $p<0.05$ was considered significant.

\section{Results}

Lesser pericyte coverage and density in the human GM on immunohistochemistry

To determine pericyte coverage and density in the human GM vasculature relative to the cortex and white matter, we performed immunohistochemical staining of pericytes and endothelia. We used CD34 as an endothelial marker, and NG2 (chondroitin sulfate proteoglycan) and PDGFR- $\beta$ as pericyte markers like several previous investigators (Abramsson et al., 2003; Armulik et al., 2005; Dellavalle et al., 2007). These pericyte markers labeled almost all the blood vessels of the forebrain, whereas other pericyte
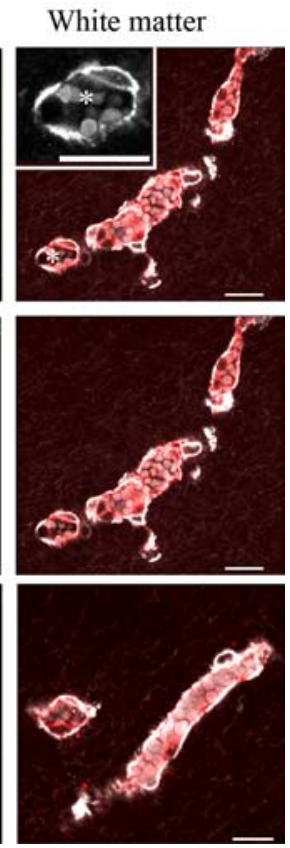

markers, $\alpha$-smooth muscle cell actin, and desmin labeled fewer blood vessels (data not shown). NG2- and PDGFR- $\beta$-positive pericytes were intimately associated with the abluminal endothelium surface in all three brain regions (cortex, white matter, and GM). We quantified pericyte coverage and density by an analysis of images acquired from triple-immunolabeled brain sections using CD34-, NG2-, and PDGFR- $\beta$-specific antibodies (Fig. 1). Pericyte density was quantified by calculating the ratio of pericytestained (NG2 or PDGFR- $\beta$ ) area and endothelium-stained (CD34) area, and pericyte coverage was assessed by measuring the overlap of the endothelial perimeter by pericytes. We found that both NG2- and PDGFR- $\beta$-positive pericyte vascular coverage were significantly less in the GM than in the cortex or white matter for all gestational age categories $(p<0.001$ all) (Fig. $2 A, B)$. Notably, in the GM, the fifth percentile cutoff value for PDGFR$\beta$-positive pericyte coverage of the blood vessels was 14.3 and $18.3 \%$, and this value for NG2-positive pericyte coverage was 31.09 and $38.4 \%$ for $17-23$ and $24-27$ weeks of gestational age, respectively (data not shown). In contrast, the corresponding fifth percentile values for the cortex or white matter were $>60 \%$ for both the gestational categories. Both NG2- and PDGFR- $\beta$-positive pericyte coverage increased in the GM as a function of gestational age $(p<0.01$ each), but not in the cortex or white matter. Consistent with the pericyte coverage, both NG2- or PDGFR- $\beta$-positive pericyte density were significantly lower in the GM than the cortex or white matter for all gestational age categories (NG2+ coverage: $p<0.001$ all comparisons, except $p=0.016$ for cortex vs GM for 23-27 weeks; PDGFR- $\beta+$ coverage: $p<0.05$ for all comparisons for 17-22 and 23-27 weeks, but $p<0.01$ for 28-35 weeks) (Fig. $2 C, D)$. In addition, PDGFR- $\beta$-positive pericyte density increased as a function of gestational age in the cortex, white matter, and GM $(p<0.0 .01,0.001$, and 0.03, respectively; ANOVA). However, NG2-positive pericyte density increased with gestational age only in the GM ( $p<0.03$, ANOVA), but not in the cortex or white matter. We did not find any detachment or looseness of pericytes around the blood vessel. Diameters of the blood vessels in the three brain regions were comparable. Together, both pericyte coverage and density were less in the GM than the cortex or white matter, which suggested that GM vasculature may be in the process of remodeling and maturation.

Fewer pericytes in human and rabbit GM than cortex or white matter on ultrastructural analysis

To further confirm the pericyte density in the GM compared with the cortex and white matter, we performed electron microscopy on 12 postmortem brain samples from fetuses and premature infants (20-28 week gestation) as well as on 12 premature rabbit 
pups (E29). Ultrastructural analysis of human samples showed that pericytes were abundant in the cerebral vasculature. Adluminal endothelial cells were connected by mature tight junctions and were separated from pericytes by basal lamina (Fig. $3 A$ ). The vascular endothelia were well invested by pericytes, and there was no evidence of pericyte detachment or loosening. The pericytes did not exhibit any difference in morphology in the GM vasculature compared with the cortex or white matter. To quantify the presence of pericytes in the vasculature, we modified the previously used classification (Lindahl et al., 1997) as follows: a, naked endothelial tube; b, pericyte cytoplasmic process present (identification of thin and scanty pericyte processes might be missed leading to an underestimation of pericyte density); c, microvessels with pericyte sectioned through nucleus; and d, not possible to classify in any of the above categories. We analyzed the three brain regions (cortex, white matter, and $\mathrm{GM}$ ) for presence or absence of pericytes (category "a" vs category "b" plus "c," respectively). Because the three brain region comparison was significant $\left(p<0.001, \chi^{2}\right.$ test $)$, we made additional pairwise comparison between the GM and the cortex or white matter. Notably, we found significantly lesser numbers of pericytes in the GM compared with those of the cortex or white matter $(p<0.001$ each, level of significance with Bonferroni's correction is 0.017 ) (Table 1, Fig. $3 A, B$ ). Consistent with the human data, the ultrastructural analysis of greater resolution rabbit pup brain (E29) images revealed a significantly lower number of pericytes in the GM than the cortex or white matter $(p<0.001$; level of significance with Bonferroni's correction is 0.017 ) (Table 1, Fig. 3C,D). Because morphology of pericyte nuclei reliably reveals the identification of pericytes, we also compared the GM with the cortex or white matter for presence or absence of pericyte nuclei and found significantly fewer pericyte nuclei in the GM than the cortex or white matter both in rabbits and humans $(p<0.05$ all $)$. There was no difference in the morphology of the pericytes among the three brain regions. Together, pericytes were fewer in both human and rabbit GM than the other brain regions.

\section{Effect of angiogenic inhibition on rabbit pericyte density and} coverage in the GM

Because high VEGF levels and rapid angiogenesis in the GM (Ballabh et al., 2007) corresponded to its lower pericyte density and 0.001 for white matter versus GM.
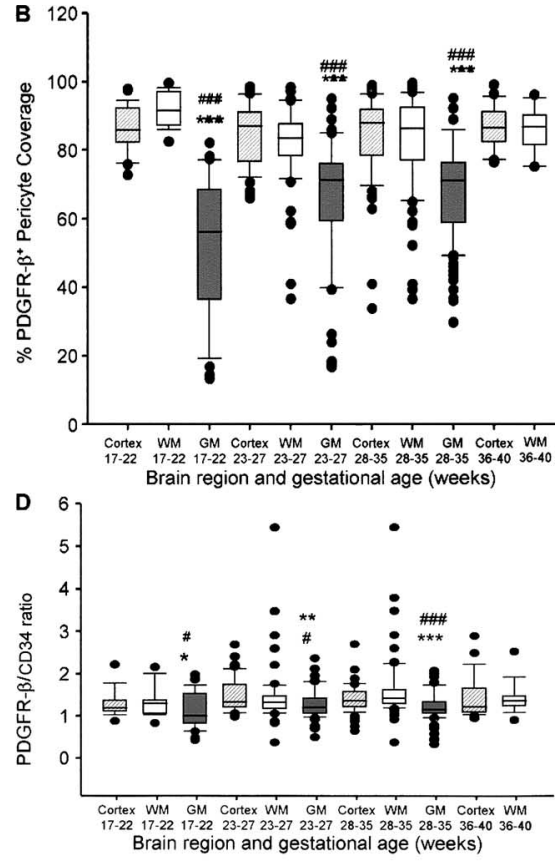

Figure 2. Pericyte coverage and density in the human GM, cortex, and white matter vasculature. $\boldsymbol{A}$, NG2-positive pericyte coverage (over pericyte on endothelium) along the outer margin of endothelium (CD34) was measured in mages acquired from cryosections of the GM, cortex, and white matter (WM). Data are shown as box-and-whisker plot that depicts median, 10th, 25th, 75th, and 90th percentile 作 cortex or white matter for all gestational age categories. In addition, pericyte coverage increased in the GM as a function of gestational age, but not in the cortex or white matter $(p<0.01) .{ }^{* *} p<0.001$ for cortex versus GM; ${ }^{\# \# \# p} p<0.001$ for white matter versus GM. $\boldsymbol{B}$, PDGFR- $\beta$-positive pericyte was measured in the images acquired from the immunostained sections of the GM, cortex, and white matter. Data are shown as box-and-whisker plot that depicts median, 10th, 25th, 75th, and 90th percentile as vertical boxes with error bars ( $n=$ outliers. PDGFR- $\beta$-positive pericyte vascular coverage was significantly lower in the GM than the cortex or white matter for all gestational age categories. In addition, pericyte coverage increased in the GM as a function of gestational age, but not in the cortex or white matte calculating the ratio of NG2-stained area and CD34-stained area. Data are shown as box-and-whisker plot that depicts median, 10th, , cortex or white matter for all gestational age categories. NG2-positive pericyte density increased with gestational age only in the GM, but not in the cortex or white matter. ${ }^{* *} p<0.01$; ${ }^{* * *} p<0.001$ for cortex versus GM; ${ }^{\# \# \# p} p<0.001$ for white matter versus GM. D, PDGFR- $\beta$-positive pericyte density was measured by calculating the ratio of PDGFR- $\beta$-stained area and CD34-stained area. Data are shown as box-and-whisker plot that depicts median, 10 th, 25 th, 75 th, and 90 th percentile as vertical boxes with error bars $(n=349,358$, and 470 was lower in the GM than the cortex or white matter for all gestational age categories. PDGFR- $\beta$-positive pericyte density increased with gestational age only in all three brain regions. ${ }^{*} p<0.05,{ }^{* *} p<0.01$, and ${ }^{* * *} p<0.001$ for cortex versus GM; ${ }^{\#} p<0.05$, and ${ }^{\# \# \#} p<$

coverage, we asked whether suppression of angiogenesis by the prenatal use of ZD6474 (VEGFR2 inhibitor) and celecoxib (COX-2 and VEGF inhibitor) in pregnant rabbits increased the pericyte coverage and density in the GM vasculature of premature pups. To this end, we used timed pregnant rabbits $(n=9)$, which were sequentially assigned to receive either oral celecoxib $\left(20 \mathrm{mg} \cdot \mathrm{kg}^{-1} \cdot\right.$ dose $\left.^{-1}\right)$ twice daily, ZD6474 $(20 \mathrm{mg} / \mathrm{kg})$ once daily, or no treatment on days 26, 27, and 28 of gestation (term, $32 \mathrm{~d}$ ). We performed C-section and delivered the pups prematurely on day 29 and killed the pups at $2 \mathrm{~h}$ age after stabilizing them. Cryosections from pup forebrain were double stained with NG2 and PECAM-1 antibodies, and acquired images were analyzed for pericyte vascular coverage and density (Fig. 4A). Pericyte vascular coverage and density in normal control rabbit pups, as in humans, were significantly lower in the GM than the cortex or white matter (coverage, $p<0.01$ and 0.05 , respectively; den- 
A

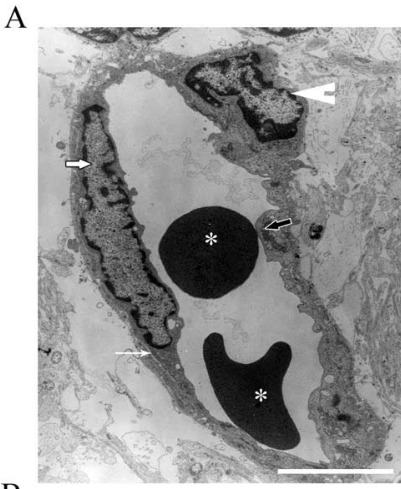

B

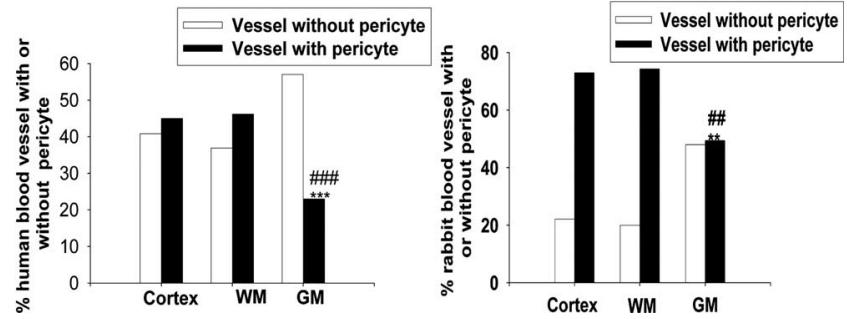

Figure 3. Fewer pericytes in GM than in the cortex or white matter (WM) on ultrastructural analysis. $\boldsymbol{A}$, Electron micrograph of cerebral microvessel in the white matter of a 20 week fetus. Expanded vascular lumen contains red blood cells (asterisks). Endothelial cells (open block arrow) line the lumen and are closed by normal interendothelial tight junctions (solid block arrows). Pericyte with a nucleus (arrowhead) is separated from the endothelium by basal lamina (arrow). Scale bar, $4 \mu \mathrm{m}$. B, Pericytes are fewer in the human GM than the cortex or white matter. Data are presented as percent blood vessels with or without pericytes in the three brain regions, cortex, white matter, and GM ( $n=12$ postmortem brain samples). Pericytes are significantly lower in number in the GM than the cortex or white matter. $\chi^{2}$ test was used for three brain region comparison, and Fisher's exact text was used for two group comparison. The level of significance after Bonferroni's correction was $0.017 .{ }^{* * *} p<0.001$ for cortex versus GM; $\# \#$ \#\# 0.001 for white matter versus GM. C, Electron micrograph of microvessel in the cerebral white matter of a premature rabbit pup of $29 \mathrm{~d}$ gestational age. Lumen of the microvessel is empty. Endothelial cells (open block arrow) lining the lumen form tight junction (solid block arrows). Pericyte with a nucleus (arrowhead) is separated from the endothelium by basal lamina (arrow). Scale bar, $4 \mu \mathrm{m}$. D, Pericytes are fewer in the rabbit GM compared with the cortex or white matter. Data are presented as percent blood vessels with or without pericytes in the three brain regions, cortex, white matter, and GM ( $n=12$ rabbit pups from 4 different litters). $\chi^{2}$ test was used for the comparison between three brain regions, and Fisher's exact text was used for two group comparison. The level of significance after Bonferroni's correction was 0.017 ${ }^{* *} p<0.001$ for cortex versus GM; ${ }^{\# \#} p<0.001$ for white matter versus GM.

sity, $p<0.01$ and 0.04 , respectively) (Fig. $4 B, C$ ). Importantly, celecoxib or ZD6474 treatment significantly enhanced the pericyte coverage in the GM ( $p<0.001$ and 0.01 , respectively) (Fig. $4 B$ ). The pericyte density was also increased with angiogenic inhibition, but was not statistically significant (Fig. 4C). Intriguingly, the pericyte coverage, although augmented with angiogenic inhibition by celecoxib or ZD6474, remained significantly lower in the GM than the cortex or white matter ( $p<0.01$ each). Likewise, the pericyte density in the GM was significantly lower in ZD6474-treated pups ( $p<0.01$ each) and substantially lower in celecoxib-treated pups than the cortex or white matter. We next evaluated the effect of angiogenic inhibition on the vascular density (number of vessels per square millimeter) and percent blood vessel area (percentage of area occupied by the blood vessels) in the three brain regions. We noted that ZD6474 treatment significantly reduced both the vascular density and the percent blood vessel area in all the three brain regions ( $p<0.001$ each) (Fig. 5). However, celecoxib decreased percent blood vessel area only in the GM $(p<0.001)$, not in the cortex and white matter. Vascular density was suppressed by celecoxib in the GM and white matter $(p<0.05$ each), but not in the cortex. This decline in vascularity on antiangiogenic treatment has been observed by other investigators (Shaheen et al., 2001; Gee et al., 2003) as well. In conclusion, rabbit GM vasculature had lesser pericyte coverage and density than the other brain regions, similar to humans, and angiogenic inhibition, although significantly increasing the perivascular coverage, did not completely normalize it to those of in the cortex or white matter. Moreover, angiogenic inhibition attenuated the vascular density, particularly of the GM.

\section{Growth factors recruiting pericytes in human fetuses and premature infants}

During development, the critical process of pericyte recruitment is regulated by molecular pathways, including PDGF-B/PDGF receptor- $\beta$, ANGPT-1/Tie-2, TGF- $\beta$ /ALK-1 and ALK-5, S1P/ $\mathrm{S} 1 \mathrm{P}$ receptor-1, and $\mathrm{N}$-cadherin/pericyte interaction (von Tell et al., 2006). Therefore, to further elucidate the mechanism underlying lesser pericyte recruitment in the GM vasculature, we compared the levels of these growth factors and their receptors among the GM, cortex, and white matter. We described previously that Ang-1 and Tie-2 levels are comparable among these three brain regions (Ballabh et al., 2007).

\section{PDGF-B and PDGFR- $\beta$ comparable in brain regions}

Western blot analysis on human brain samples revealed that the expression of PDGF-B protein levels was similar in the three brain regions (cortex, white matter, and GM) and that it displayed an insignificant downward trend with the increasing gestational age (Fig. 6A). Accordingly, gene expression of PDGF-B, measured by real-time PCR, showed no significant difference in the expression of PDGF-B among the three brain regions (Fig. $6 C)$. Notably, PDGF-B mRNA levels decreased significantly as a function of gestational age in all the three regions ( $p<0.05$ each) (Fig. 6C). We next evaluated protein and gene expression of PDGFR- $\beta$ receptor in human samples. We found that PDGFR- $\beta$ protein expression trended to be higher in the GM than the cortex or white matter (Fig. 6B). However, this comparison was not statistically significant. Of note, PDGFR- $\beta$ protein level was lower in older premature infants (28-35 weeks) compared with younger premature infants (23-27 weeks) in all the three regions $(p<0.05$ each). Likewise, PDGFR- $\beta$ gene expression appeared to be greater in the GM than the other brain regions, but the comparison was not significant $(p=0.1)$. Moreover, it did not alter significantly with the advancing gestational age (Fig. 6D). Evaluation of double immunostaining of cryosections with PDGFR- $\beta$ - and CD34-specific antibody showed that the immunoreactivities to PDGFR- $\beta$ were associated with the blood vessels as well as with the radial glia in the GM, which is consistent with the previous reports (Maxwell et al., 1998). However, PDGFR- $\beta$ was expressed predominantly in the blood vessels of the cortex and white matter and weakly in the glial fibers of the white matter of only younger gestational age subjects ( $<20$ weeks). The glial expression of PDGFR- $\beta$ was confirmed by double labeling of the brain sections with GFAP- and PDGFR- $\beta$-specific antibodies (data not shown). Although PDGFR- $\beta$-positive pericytes were fewer in the GM than other brain regions, PDGFR- $\beta$ protein levels tended to be higher in the GM than the other brain regions. This could be ascribed to its expression in the radial glia of the GM in addition to the blood vessels. Hence, expression of PDGF-B and PDGFR- $\beta$ were comparable in the three brain regions; and PDGFR- $\beta$ was expressed both in the radial glia and blood vessels of the GM as well as in the vasculature of the cortex and white matter. 
Table 1. Pericytes in the vasculature of GM, cortex, and white matter

\begin{tabular}{|c|c|c|c|c|c|c|}
\hline \multirow[b]{2}{*}{ Subjects } & \multirow[b]{2}{*}{ Brain regions } & \multirow[b]{2}{*}{$n$} & \multicolumn{4}{|l|}{ Category } \\
\hline & & & $a$ & b & c & $d$ \\
\hline \multirow[t]{3}{*}{ Human } & Cortex & 120 & $49(40.8)$ & $30(25)$ & $24(20)$ & $17(14.2)$ \\
\hline & White matter & 119 & $44(36.9)$ & $34(28.5)$ & $21(17.6)$ & $20(16.8)$ \\
\hline & GM & 200 & $114(57)$ & $25(12.5)$ & $21(10.5)$ & $40(20)$ \\
\hline \multirow[t]{3}{*}{ Rabbit } & Cortex & 122 & $27(22.1)$ & 49 (10.1) & $40(32.7)$ & $6(4.9)$ \\
\hline & White matter & 105 & $21(20)$ & $49(46.7)$ & $29(27.6)$ & $6(5.7)$ \\
\hline & GM & 281 & $135(48)$ & $73(25.9)$ & $66(23.5)$ & $7(2.5)$ \\
\hline
\end{tabular}

$n$ refers to the number of blood vessels evaluated in a brain region. The number in parentheses indicates percentage. Categories indicate the following: $a$, naked endothelial tube; $b$, pericyte cytoplasmic process present (identification of thin and scanty pericyte processes might be missed, leading to an underestimation of pericyte density); c, microvessels with pericyte sectioned through nucleus; and d, not possible to classify in any of the above categories. Comparison of the presence of pericytes (category "a" vs category" $\mathrm{b}^{\prime \prime}$ plus $\mathrm{c}^{\prime \prime}$ ) among the blood vessels of cortex, white matter, and GM was significant $\left(p<0.001, \chi^{2}\right.$ test) in both rabbits and humans. Pericyte density is lower in the GM than the cortex and white matter both in rabbits and humans ( $p<0.001$ all, Fisher's exact test; level of significance with Bonferroni's correction is 0.017$)$. In addition, a comparison of presence (category " $c$ ") or absence of pericytic nucleus (category "a" plus "b") between GM and cortex or white matter was also statistically significant (all $p<0.05$ ).

TGF- $\beta 1$, ALK-1, and ALK-5

Because TGF- $\beta 1$ induces differentiation of precursor cells into pericytes and inhibits endothelial proliferation (von Tell et al., 2006), we next evaluated the expression of TGF- $\beta 1$ and its receptor ALK- 1 and ALK-5 in the cortex, white matter, and GM. To this end, we immunolabeled coronal brain sections with TGF- $\beta 1$ - and CD34-specific antibodies. We found that TGF- $\beta 1$ immunoreactivity was abundant in both blood vessels and the surrounding neural structures of the cortex and white matter; but diminished in the neural structure with advancing gestational age $(>20$ weeks) (Fig. 7A). In the GM, TGF- $\beta 1$ expression was either weak or absent in the vasculature and scarce in the neural structures. Double immunolabeling with GFAPand TGF- $\beta 1$-specific antibodies revealed that TGF- $\beta 1$ was coexpressed only in some of the GFAP+ radial glia of the GM and a few glial fibers of the white matter at younger gestational ages (16-20 weeks). Expression of TGF- $\beta 1$ in neural structures, in addition to the blood vessels, is consistent with the reports of other investigators (Montuenga et al., 1998). Western blot analysis showed that TGF- $\beta 1$ protein levels were significantly lower in the GM than the cortex or white matter for all subjects together (Fig. $7 B)(p<$ 0.023 and 0.01 , respectively). In addition, it did not change significantly with increasing gestational age. Of note, in contrast to the lower TGF- $\beta 1$ protein level in the GM, its gene expression was significantly higher in the GM than the cortex $(p<0.005)$ (Fig. 7C), but not the white matter $(p=0.1)$, in a combined analysis of all subjects $(16-40$ weeks; $n=24)$. This comparison was also significant for
NG2 PECAM-1

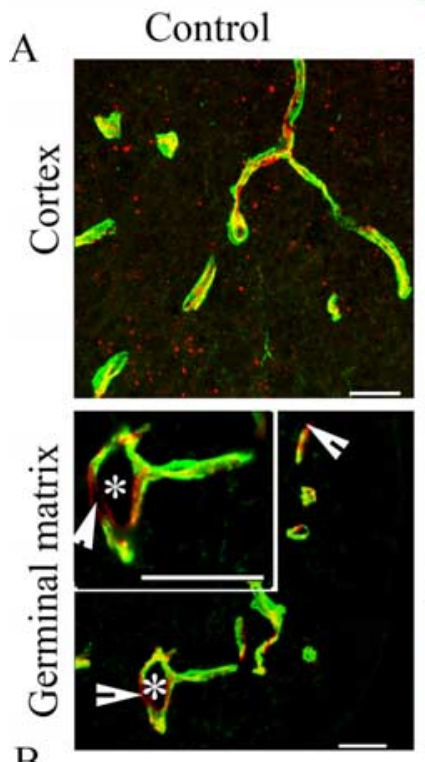
ZD6474

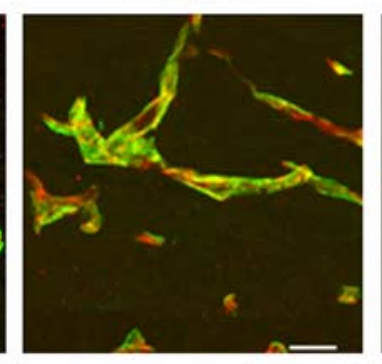

Celecoxib
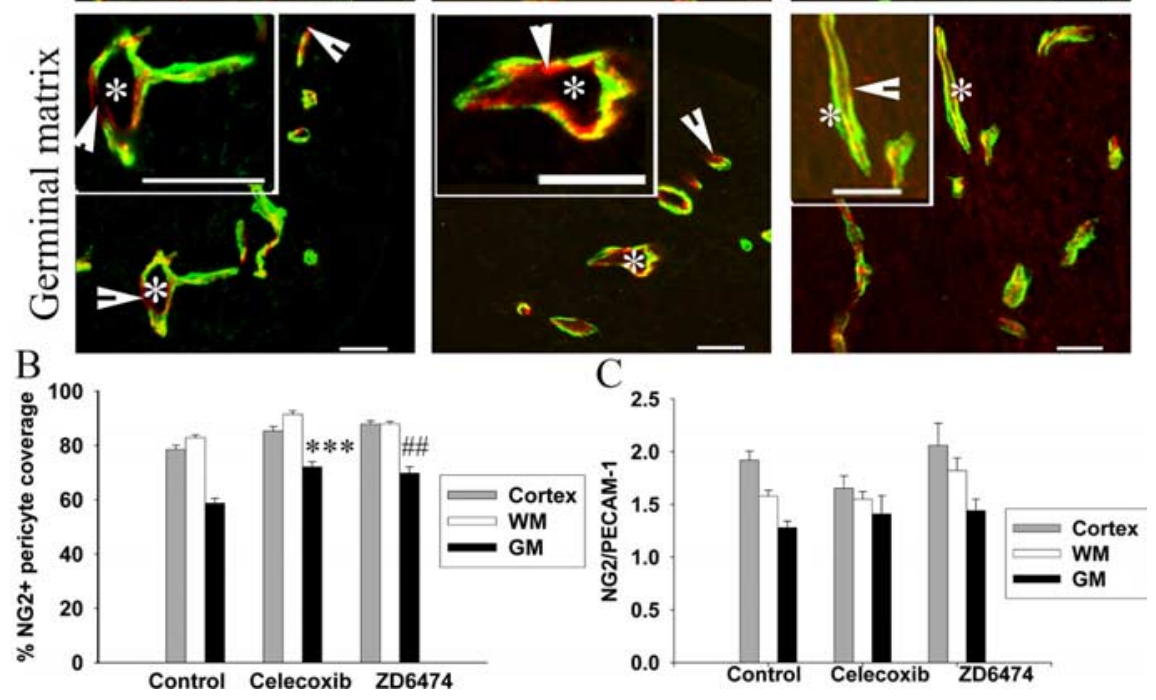

Figure 4. Angiogenic inhibition increases pericyte coverage in rabbit pups. $A$, Coronal cyrosections from the rabbit cortex and GM were stained with NG2- and PECAM-1-specific antibodies. In both GM and cortex, NG2-positive pericytes were closely associated with the endothelia. Coverage of the endothelia by NG2-positive pericytes was incomplete (arrowhead) and less in the GM than observed in cortex. The insets show a GM blood vessel under high magnification that has less pericyte coverage. The asterisk indicates vessel shown under high magnification. Note vascular coverage by NG2-positive pericytes in the GM was greater in pups treated with angiogenic inhibitors, celecoxib or ZD6474, than controls. Scale bar, $20 \mu \mathrm{m}$. B, NG2-positive pericyte coverage was compared among celecoxib- and ZD6474-treated pups as well as untreated controls in the three brain regions. Data are mean \pm SEM $(n=52,50$, and 63 blood vessels from the cortex, white matter, and GM, respectively, for untreated controls, and pups treated with celecoxib or ZD6474; 5 pups in each group). Pericyte vascular coverage in untreated controls was less in the GM than the cortex or white matter $(p<0.01$ and 0.05$)$ as well as in celecoxib- and ZD6474-treated pups ( $p<0.01$ each). Celecoxib or ZD6474 treatment significantly enhanced the perivascular coverage by pericytes in the GM. ${ }^{* * *} p<0.001$ control versus celecoxib treatment for GM; ${ }^{\# \#} p<0.01$ control versus ZD6474 treatment for GM. C, NG2-positive pericyte density was compared among celecoxib- and ZD6474-treated pups as well as untreated controls in the three brain regions. Data are mean $\pm \operatorname{SEM}(n=52,50$, and 63 blood vessels from the cortex, white matter, and GM, respectively, in untreated controls, and pups treated with celecoxib and ZD6474; 5 pups in each group). Pericyte vascular density was significantly less in the GM than the cortex or white matter in untreated controls. The pericyte density did not significantly increase with the celecoxib or ZD6474 treatment.

fetuses (17-22 weeks) and older premature infants (28-35 weeks) (within group analysis). These data suggest that TGF- $\beta 1$ protein may be subject to increased turnover in the GM. 


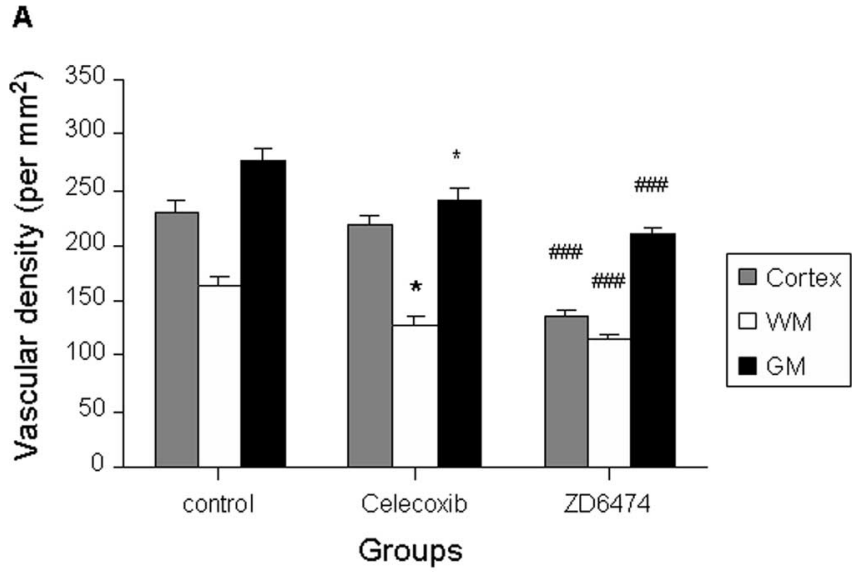

B

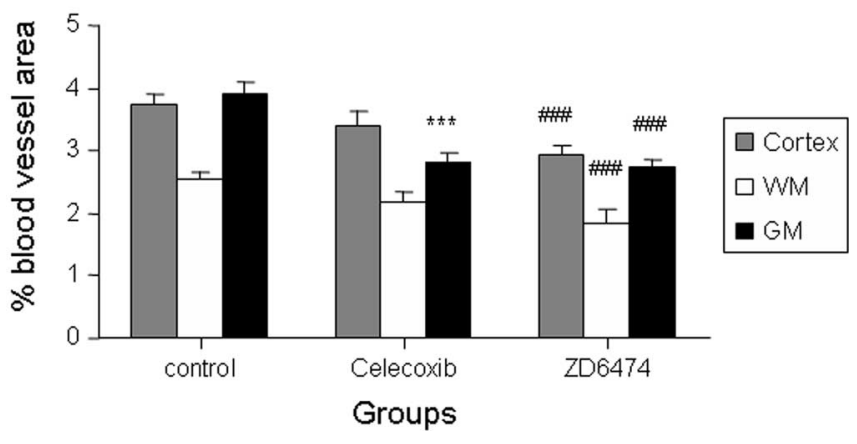

Figure 5. ZD6474 suppressed vascularity in all brain areas, but celecoxib primarily in the GM Images from rabbit brain cyosections immunostained with PECAM-1-specific antibody were evaluated for vessel density (per square millimeter) and percent blood vessel area. Data are mean \pm SEM ( $n=45$ blood vessels from each area, cortex, white matter, and GM, in untreated controls; similar number of blood vessels from pups treated with celecoxib and ZD6474; 5 pups in each group). ZD6474 significantly reduced both the blood vessel density $(\boldsymbol{A})$ and percent blood vessel areas (B) in the three brain regions (GM, cortex, and white matter). Celecoxib significantly reduced blood vessel density only in the $G M$, but not in the other two brain regions. It diminished percent blood vessel area in the GM and white matter, but not in the cortex. ${ }^{*} p<$ 0.05 , and ${ }^{* * *} p<0.001$ for regional comparison between control and celcoxib treatment; and $\#$ \#\#\# $<0.001$ for regional comparison between control and ZD6474 treatment.

Two TGF- $\beta$ type 1 receptors, ALK-5 and ALK-1, are indispensable for angiogenesis as demonstrated by defect in vessel formation in mouse embryo deficient in either of them. Double immunolabeling of cryosections with ALK-1-specific antibody showed that it was expressed in all the blood vessels of GM, cortex, and white matter, and endothelial ALK-1 immunoreactivities colocalized with CD34 (Fig. 8A). The gene expression of ALK-1, measured by real-time PCR, was similar among the three brain regions and did not change significantly with the increasing gestational age (Fig. $8 \mathrm{~B}$ ).

Double immunolabeling of coronal brain sections revealed that ALK-5 was expressed in the larger blood vessels of cortex, white matter, and the GM (Fig. 9A). Expression in smaller blood vessels were either weak or absent. In addition, immunoreactivities to ALK-5 attenuated with increasing gestational age. Accordingly, Western blot analysis showed an insignificant downward trend in ALK-5 protein levels with advancing gestational age (Fig. $9 B)$. Moreover, ALK-5 expression was comparable in the GM, cortex, and white matter. Of note, ALK-5 gene expression decreased significantly with increase in gestational age in the cortex, white matter, and GM ( $p=0.005,0.009$, and 0.03, respectively) (Fig. 9C). Intriguingly, ALK-5 gene expression was significantly greater in the GM than in the white matter $(p<0.03)$, but not in the cortex. In conclusion, a significant decline in the gene expression of ALK-5 with increasing gestational age and higher level of expression in the GM relative to other brain areas suggest that ALK-5 might play key roles in pericyte recruitment around the immature blood vessels of the GM and that it has lower expression in the brain compartments with quiescent and mature vasculature.

\section{S1P1 and N-cadherin enriched in the GM}

$\mathrm{S} 1 \mathrm{P} 1$ has critical role in angiogenesis and mice deficient in S1P1 receptor exhibit failure of pericyte recruitment around the endothelial tubes leading to hemorrhages throughout their bodies and death at E12.5 (Liu et al., 2000; Spiegel and Milstien, 2003; Tillet et al., 2005). Notably, S1P1 regulates N-cadherin-mediated endothelial pericyte interaction and a lack of $\mathrm{N}$-cadherin is attended by impaired pericyte covering around the endothelial outgrowths. Therefore, we evaluated S1P1 and N-cadherin in the GM, cortex, and white matter of fetuses and premature infants. We found that S1P1 protein levels are significantly elevated in the GM than the cortex or white matter in both fetuses and premature infants (Fig. 10A) $(p=0.014$ and 0.01 , respectively). In addition, its level increased as a function of gestational age in the three brain regions ( $p<0.05$ each). Similarly, gene expression of S1P1 was greater in the GM than the cortex and white matter (Fig. $10 C)(p=0.02$ and 0.01 , respectively, for fetuses plus premature infants). In addition, S1P1 mRNA expression showed an insignificant trend to increase with gestational age. We next evaluated $\mathrm{N}$-cadherin in the three brain regions and found that $\mathrm{N}$-cadherin protein levels showed an insignificant trend of greater expression in the GM than the cortex and white matter. N-cadherin protein levels showed a downward pattern with the advancing gestational age. $\mathrm{N}$-cadherin gene expression was greater in the GM than the cortex and white matter ( $p<0.012$ and 0.001 , respectively) (Fig. $10 \mathrm{~B})$ for an analysis of all subjects combined (16-40 week weeks, $n=24)$. In addition, it decreased significantly in all areas as a function of gestational age ( $p<0.05$ each) (Fig. 10D). Together, both $\mathrm{N}$-cadherin and S1P1 were substantially greater in the GM than the cortex or white matter; and S1P1 exhibited an upward trend and, conversely, $\mathrm{N}$-cadherin a downward trend with increasing gestational age.

\section{Discussion}

As both the birth rate (Shennan and Bewley, 2006) and survival of the premature infants have increased (Tommiska et al., 2001), GMH-IVH and resultant neurologic sequelae including cerebral palsy, posthemorrhagic hydrocephalus, and mental retardation have developed into major public health concerns. In this study, we evaluated morphologic aspects and molecular mechanisms involved in the pathogenesis of GMH based on the premise that there is rapid angiogenesis prevailing in the GM and that the nascent blood vessels have fewer pericytes to provide structural integrity. In the present study, predictably, we observed that pericytes were diminished in the GM vasculature of human fetuses, premature infants as well as in premature rabbit pups, and that angiogenic inhibition increased the pericyte coverage of rabbit GM microvasculature. We also found distinct differences in the expression patterns of some factors responsible for recruiting pericytes in the GM compared with the cortex or white matter. Specifically, TGF- $\beta 1$ protein expression was lower, whereas S1P1 and $\mathrm{N}$-cadherin levels were higher in the human GM than the cortex or white matter.

The most important and novel observation made in this study 
was that both pericyte coverage and density were reduced in the GM compared with the cortex or white matter. Several lines of evidence suggest that pericytes stabilize blood vessels and provide structural integrity. First, PDGF-B-deficient mouse embryos lack capillary pericytes and develop numerous capillary microaneurysms in the cerebral vasculature that rupture in late gestation (Lindahl et al., 1997). Second, metabolic injury to pericytes in diabetes mellitus is associated with retinal microaneurysm formation and hemorrhage (Kern and Engerman, 1996), and proliferative retinopathy develops with pericyte density of $<50 \%$ of normal in PDGF-Bdeficient mice (Enge et al., 2002). Third, pericyte degeneration is seen in hereditary cerebral hemorrhage with amyloidosis (Verbeek et al., 1997). Fourth, pericytes play roles in tight junction formation, bloodbrain barrier differentiation, and formation of basal lamina (Balabanov and Dore-Duffy, 1998). Thus, pericytes exhibit unique functional characteristics that seem to be decisive for the structural support of blood vessels, and a threshold density of pericytes might be critical for cerebral vessel integrity and function. The present study showed low pericyte coverage in the GM vasculature compared with the other brain regions; and $5 \%$ of blood vessel had $<20 \%$ PDGFR- $\beta$-positive and $<40 \%$ NG2-positive pericyte coverage in the GM compared with $>60 \%$ in the cortex and white matter of all premature infants of $<28$ week gestational age (Fig. 2). Thus, it is plausible that a relative lack of pericytes contributes to the fragility of GM vasculature and its propensity to hemorrhage. Because the pathogenesis of GMH is usually attributed to vascular wall weakness of the GM vasculature and fluctuation of cerebral blood flow in unstable preterm newborns, as a result of hypoxia, hypercarbia, pneumothorax, patent ductus arteriosus, chorioamnionitis, seizures, and respiratory distress syndrome (Kenny et al., 1978; Volpe, 1989; Antoniuk and da Silva, 2000), we speculate that GM blood vessels with relatively sparse pericytes rupture when challenged by a fluctuation in the cerebral blood flow.

Another key observation was that angiogenic inhibition by celecoxib and ZD6474 increased pericyte coverage around the blood vessels and reduced the vessel density. Because angiogenic inhibitors suppress VEGF levels or its receptors leading to apoptosis of endothelial cells not protected by pericytes, the increase in the pericyte coverage on celecoxib and ZD6474 treatment appears to be secondary to a selective loss of blood vessels lacking pericytes. This finding provides a mechanistic basis to our previous observation that angiogenic inhibition reduces GMH in premature rabbit pups (Ballabh et al., 2007). Importantly, pruning the GM vasculature might reduce the oxygenation of GM, which may have detrimental effects on the normal development of the GM. Therefore, we speculate that optimizing the dose of angiogenic inhibitors to stabilize the GM vasculature (greater pericyte recruitment) without pruning the functional vessels might be better strategy to prevent GMH. In an elegant experiment in orthotopic xenograft model of human glioma, it has been shown that optimization of dose of VEGFR2 blockade "normalizes" the morphology of the vasculature by enhancing pericyte coverage via upregulation of angiopoietin-1 and by degradation of pathological thick basal lamina via MMP (matrix metalloproteinase) activation (Stoeltzing et al., 2003; Winkler et al., 2004; Jain, 2005; Chantrain et al., 2006). These fortified vessels become structurally more stable and enhance oxygen delivery to tumor. Hence, VEGFR2 and COX2 inhibition needs additional evaluation in different dosing regimens to determine a treatment schedule that is efficacious in preventing IVH and safe to the brain and overall development of the premature infants.

Notably, the pericyte density and coverage, although augmented by angiogenic inhibition, remained substantially lower in the GM than the cortex or white matter. This might be attributable either to incomplete suppression of angiogenesis in the GM or to deficiency of growth factors in the GM that are responsible for pericyte recruitment. Therefore, we evaluated important ligands and their receptors that are responsible for recruiting pericytes to the vasculature during angiogenesis. However, we did not evaluate Tie-2 and ANGPT in this study because we have already published the data that expression of Tie- 2 and angiopoietin- 1 are comparable in the GM, cortex, 
A
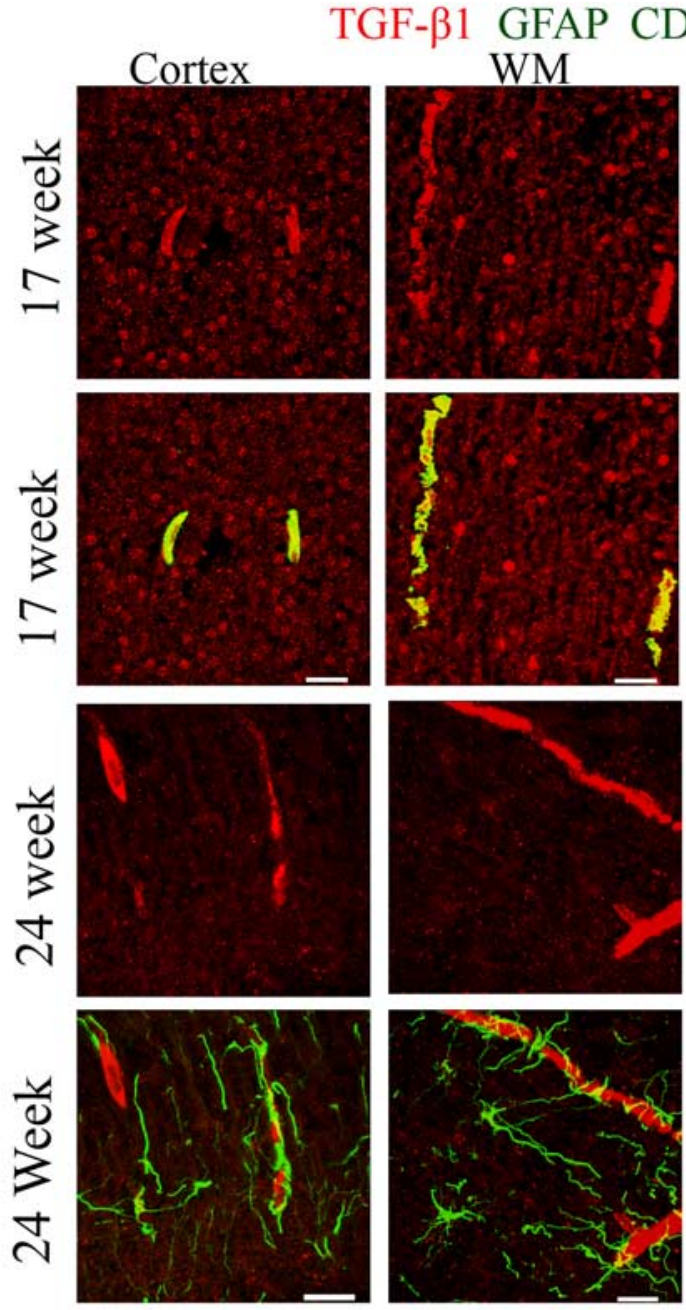
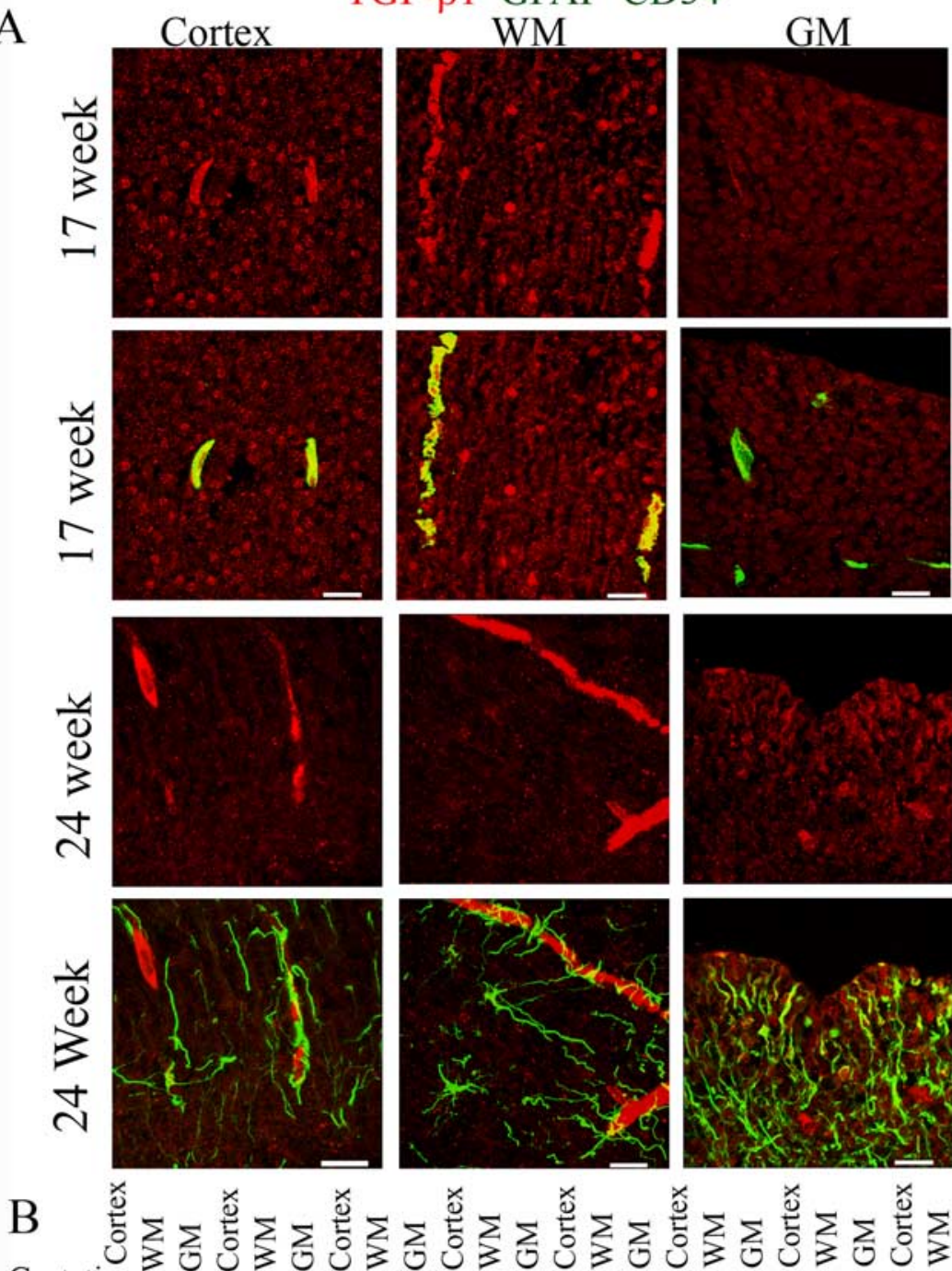

Gestation 16 (week$$
\text { (1) }
$$

$$
\text { TGF } \beta 1(13 \mathrm{kDa})
$$
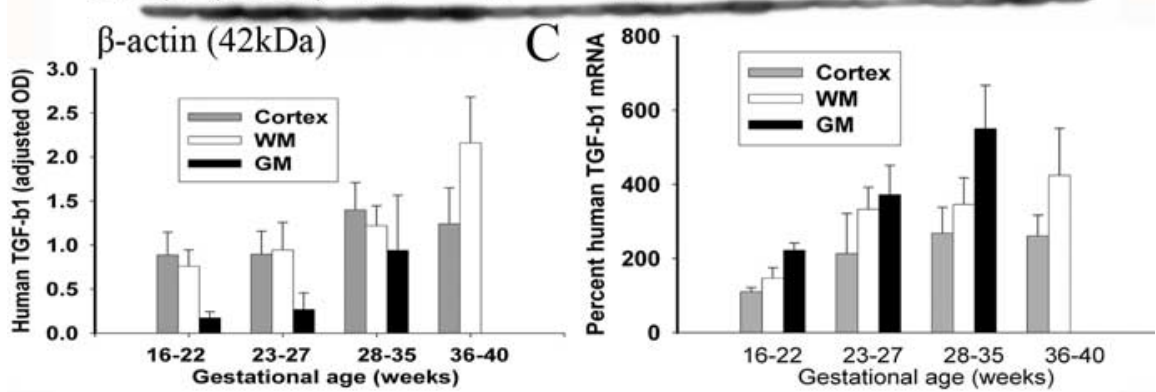

Figure 7. TGF- $\beta 1$ levels lower in the GM than the cortex or white matter. $\boldsymbol{A}$, The coronal brain section of a 17 week fetus was double immunostained with TGF- $\beta 1$ and CD34 antibodies. The expression of TGF- $\beta 1$ in the GM blood vessels was either weak or absent. It was also weakly expressed in the neural structures of the GM. In contrast, in the cortex and white matter, it was strongly expressed in blood vessels and surrounding neural structures. Double labeling of a frontal lobe sections of a 24 week premature infant with TGF- $\beta 1$ and GFAP antibodies showed that TGF- $\beta 1$ was expressed in few GFAP + radial glia of the GM and some glial fibers of the white matter. Scale bar, 20 $\mu \mathrm{m} . \boldsymbol{B}$, Representative Western blot analysis of TGF- $\beta$ in the GM, cortex, and white matter of eight human subjects. Data are mean \pm SEM ( $n=24$; fetuses, premature plus mature infants). TGF- $\beta$ was significantly less in the GM (arrowhead) than the cortex or white matter for all subjects together ( $p<0.023$ and 0.01 , respectively). It did not change insignificantly with increasing gestational age. C, TGF- $\beta$ mRNA levels were measured by real-time $P C R$ in human subjects ( $n=24$; fetuses, premature plus mature infants). TGF- $\beta$ mRNA levels were higher in the GM than the cortex ( $p<0.005$ ) but not the white matter $(p=0.1)$, for an analysis of all subjects together. TGF- $\beta$ gene levels did not change significantly with the advancing gestational age. and white matter (Ballabh et al., 2007). Importantly, rapid angiogenesis in the GM, induced by the higher levels of ANGPT-2 and VEGF (Ballabh et al., 2007), corresponded with reduced pericyte density and coverage in this subventricular zone (this study). This association of accelerated angiogenesis and few pericytes also has been observed by several investigators in tumor vasculature (Stratmann et al., 1998; Koga et al., 2001; Zhang et al., 2003). Hence, high VEGF in collaboration with angiopoietin-2 is likely to initiate formation of immature blood vessels in the GM that lack pericytes.

Pericytes play critical role in angiogenesis. They can originate from multiple sources during embryonic development including local and distal mesenchyme (Hungerford and Little, 1999; Sartore et al., 2001). Common progenitors (Flk1+ stem cells) differentiate into either endothelial cells or pericytes in presence of VEGF or PDGF-B, respectively (Yamashita et al., 2000). In addition, fibroblast and myofibroblast can differentiate into pericytes, and bone marrow cells can be recruited around the vasculature as pericytes (Tomasek et al., 2002). Conversely, pericytes can differentiate into fibroblast and osteogenic cells (Doherty et al., 1998). Importantly, NG2-positive pericytes differentiate into cells of neural lineage in presence of basic fibroblast growth factor and express neuronal and glial cell markers (Dore-Duffy et al., 2006). Based on these considerations, we speculate that pericytes might have multiple roles in the GM, where neurogenesis, oligogenesis, and angiogenesis are prevailing during brain development.

TGF- $\beta 1$ promotes stabilization of blood vessels by differentiation of pericytes from mesenchymal cells, deposition of extracellular matrix, and assembly of pericytes around endothelia during angiogenesis $(\mathrm{Ar}-$ mulik et al., 2005). TGF- $\beta 1$ is expressed by endothelial cells as well as pericytes; and its effects are generally antiangiogenic, but could be proangiogenic depending on the context and concentration (Bertolino et al., 2005). Low concentration of TGF- $\beta 1$ stimulates endothelial 
proliferation, whereas high dose of TGF- $\beta 1$ suppresses it. It regulates angiogenesis by balancing the signaling between two receptors, ALK-1 and ALK-5. Activation of the TGF- $\beta 1 /$ ALK-5 pathway inhibits endothelial proliferation and promotes mesenchymal differentiation into pericytes, whereas the TGF- $\beta 1 /$ ALK-1 pathway induces endothelial proliferation and migration. Thus, in the present study, a low protein concentration of TGF- $\beta 1$ in the GM led us to speculate that a lower TGF- $\beta 1$ level might have proangiogenic effects and that it might be linked to lower pericyte coverage in the GM vasculature. A greater level of ALK-5 in the GM suggests that vascular maturation by recruitment of pericytes is also occurring simultaneously to endothelial proliferation. Together, GM vasculature is distinctive with respect to rapid vascular remodeling and maturation compared with the other brain compartments.

Both S1P1 and N-cadherin have critical roles in angiogenesis and mice deficient in either of them exhibit failure of pericyte recruitment around the endothelial tubes leading to cerebral hemorrhage and death (Liu et al., 2000; Spiegel and Milstien, 2003; Tillet et al., 2005). S1P1 regulates N-cadherinmediated endothelial pericyte interaction and a lack of $\mathrm{N}$-cadherin is attended by impaired pericyte coverage around the endothelial out-

growths. Thus, a higher level of these two molecules in the GM further suggests that GM vasculature is unique in the forebrain because it is enriched with blood vessels undergoing remodeling and pericyte recruitment.

In conclusion, there are several distinctive features of GM vasculature. It possesses fewer pericytes than the vasculature of other brain regions and the pericytes increase in density with angiogenic inhibition. Moreover, the GM is depleted of TGF- $\beta 1$, favoring induction of angiogenesis, and has high expression of $\mathrm{N}$-cadherin and $\mathrm{S} 1 \mathrm{P} 1$, assisting vascular maturation with recruitment of pericytes. Of note, components of the blood-brain barrier, which support vascular structural integrity, including aquaporin-4-positive astrocyte endfeet, tight junction molecules, and collagen IV in basal lamina, are comparable in the three brain regions (GM, cortex, and white matter) (Anstrom et al., 2005; Ballabh et al., 2005; El-Khoury et al., 2006). The pericyte, a major component of the blood-brain barrier, which is relatively deficient in the GM compared with cortex and white matter, may be an important determinant of the structural stability of the GM vasculature. The paucity of pericyte coverage of the GM vasculature would therefore contribute to its vulnerability to hemorrhage.
Alk-1 CD34
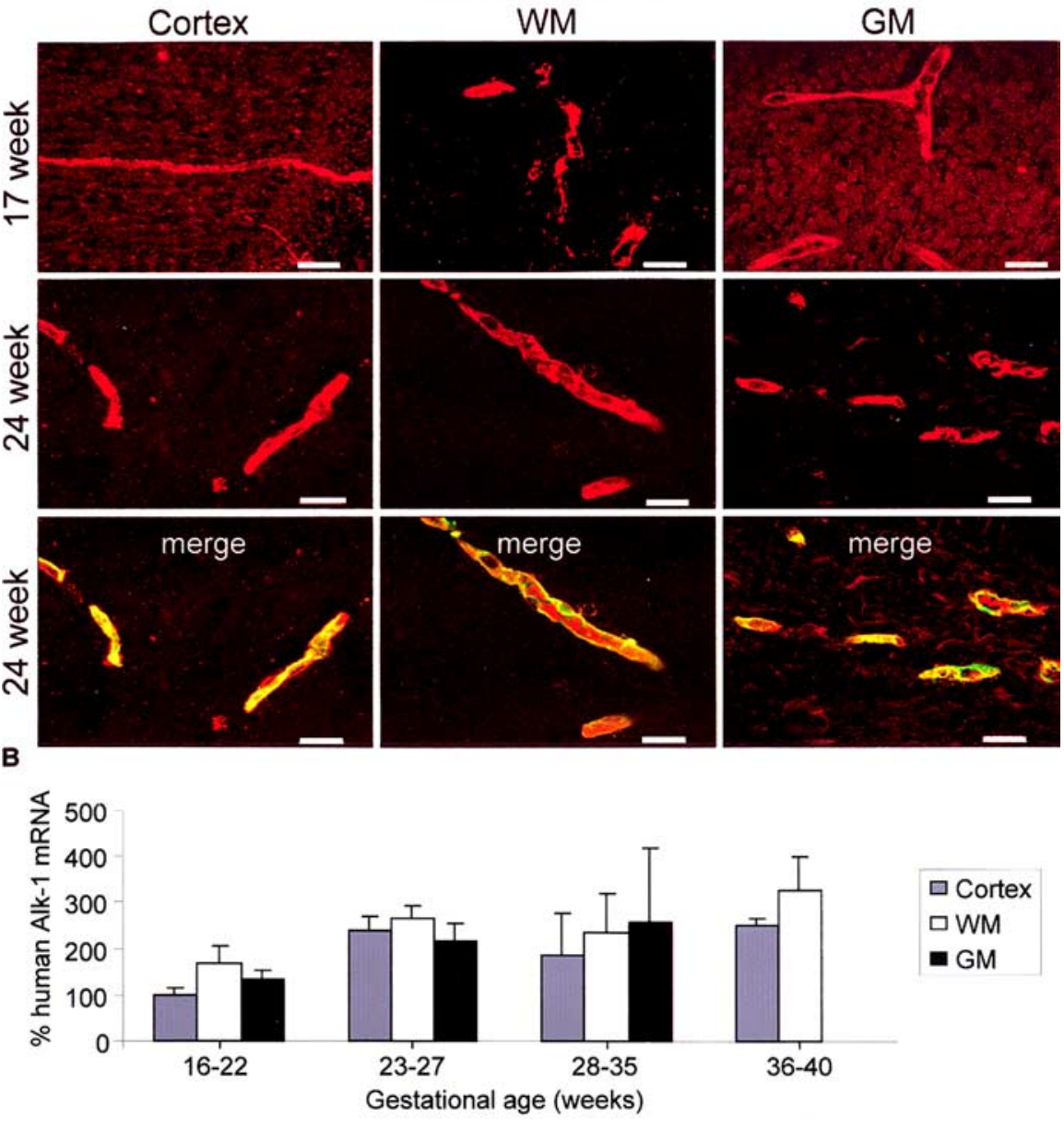

Figure 8. ALK-1 comparable in the three brain regions. $A$, Representative immunofluorescence of cryosections from 17 week fetus and 24 week premature infant labeled with ALK-1-specific antibody. It shows that ALK-1 is expressed in all the blood vessels of GM, cortex, and 24; fetuses, premature plus mature infants). ALK-1 mRNA level, measured by real-time PCR, was similar among the three brain regions and did not change significantly with the increasing gestational age.

\section{References}

Abramsson A, Lindblom P, Betsholtz C (2003) Endothelial and nonendothelial sources of PDGF-B regulate pericyte recruitment and influence vascular pattern formation in tumors. J Clin Invest 112:1142-1151.

Angevine Jr JB, Bodian D, Coulombre AJ, Edds Jr MV, Hamburger V, Jacobson M, Lyser KM, Prestige MC, Sidman RL, Varon S, Weiss PA (1970) Embryonic vertebrate central nervous system: revised terminology. Anat Rec 166:257-261.

Anstrom JA, Thore CR, Moody DM, Challa VR, Block SM, Brown WR (2005) Morphometric assessment of collagen accumulation in germinal matrix vessels of premature human neonates. Neuropathol Appl Neurobiol 31:181-190.

Antoniuk S, da Silva RV (2000) Periventricular and intraventricular hemorrhage in the premature infants. Rev Neurol 31:238-243.

Armulik A, Abramsson A, Betsholtz C (2005) Endothelial/pericyte interactions. Circ Res 97:512-523.

Balabanov R, Dore-Duffy P (1998) Role of the CNS microvascular pericyte in the blood-brain barrier. J Neurosci Res 53:637-644.

Ballabh P, Braun A, Nedergaard M (2004a) The blood-brain barrier: an overview: structure, regulation, and clinical implications. Neurobiol Dis 16:1-13.

Ballabh P, Braun A, Nedergaard M (2004b) Anatomic analysis of blood vessels in germinal matrix, cerebral cortex, and white matter in developing infants. Pediatr Res 56:117-124.

Ballabh P, Hu F, Kumarasiri M, Braun A, Nedergaard M (2005) Develop- 
A

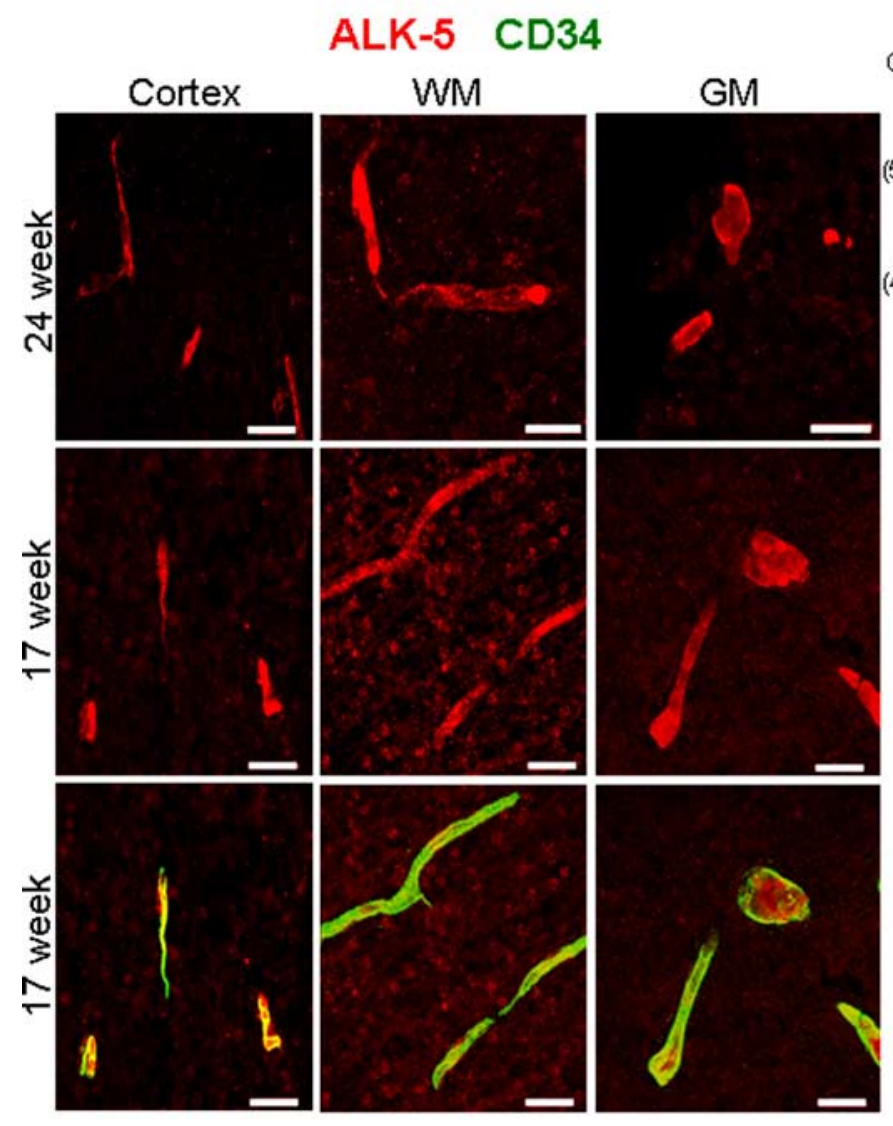

B

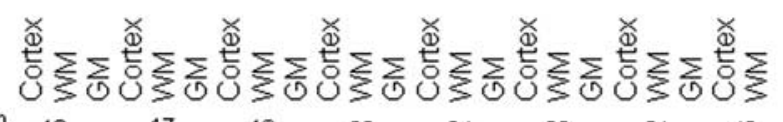

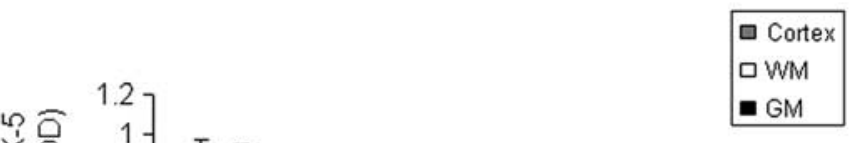
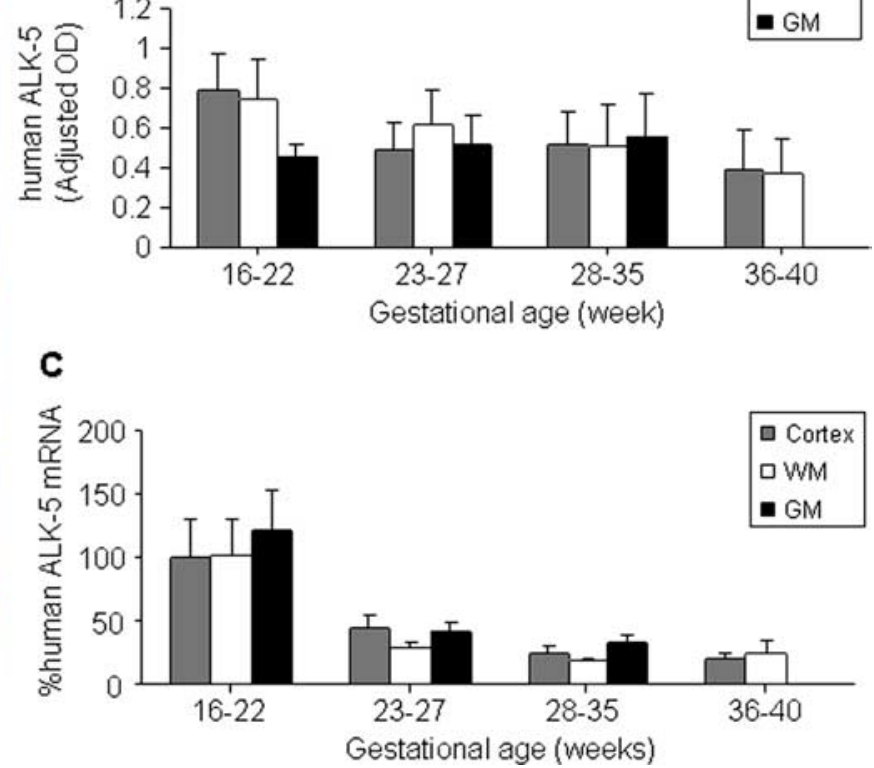

Figure 9. ALK-5 reduces with maturation. $A$, Coronal brain sections double immunolabeled with ALK-5 and CD34. It showed that ALK-5 was expressed in blood vessels and the immunosignals colocalized with CD34. Scale bar, $20 \mu \mathrm{m}$. B , Data are mean \pm SEM ( $n=24$; fetuses, premature plus mature infants). ALK-5 protein levels were comparable in the GM, cortex, and white matter, and showed insignificant downward trend as a function of gestational age. C, ALK-5 mRNA levels were measured by real-time PCR in human subjects ( $n=24$; fetuses, premature plus mature infants). ALK-5 gene expression was significantly greater in the GM than the white matter, but not the cortex. ALK-5 gene expression decreased significantly with increase in gestational age in all three brain regions.

ment of tight junction molecules in blood vessels of germinal matrix, cerebral cortex, and white matter. Pediatr Res 58:791-798.

Ballabh P, Xu H, Hu F, Braun A, Smith K, Rivera A, Lou N, Ungvari Z, Goldman SA, Csiszar A, Nedergaard M (2007) Angiogenic inhibition reduces germinal matrix hemorrhage. Nat Med 13:477-485.

Bertolino P, Deckers M, Lebrin F, ten Dijke P (2005) Transforming growth factor-beta signal transduction in angiogenesis and vascular disorders. Chest 128:585S-590S.

Chantrain CF, Henriet P, Jodele S, Emonard H, Feron O, Courtoy PJ, DeClerck YA, Marbaix E (2006) Mechanisms of pericyte recruitment in tumour angiogenesis: a new role for metalloproteinases. Eur J Cancer 42:310-318.

Dellavalle A, Sampaolesi M, Tonlorenzi R, Tagliafico E, Sacchetti B, Perani L, Innocenzi A, Galvez BG, Messina G, Morosetti R, Li S, Belicchi M, Peretti G, Chamberlain JS, Wright WE, Torrente Y, Ferrari S, Bianco P, Cossu G (2007) Pericytes of human skeletal muscle are myogenic precursors distinct from satellite cells. Nat Cell Biol 9:255-267.

Doherty MJ, Ashton BA, Walsh S, Beresford JN, Grant ME, Canfield AE (1998) Vascular pericytes express osteogenic potential in vitro and in vivo. J Bone Miner Res 13:828-838.

Dore-Duffy P, Katychev A, Wang X, Van Buren E (2006) CNS microvascular pericytes exhibit multipotential stem cell activity. J Cereb Blood Flow Metab 26:613-624.

du Plessis AJ, Volpe JJ (2002) Perinatal brain injury in the preterm and term newborn. Curr Opin Neurol 15:151-157.

El-Khoury N, Braun A, Hu F, Pandey M, Nedergaard M, Lagamma EF, Ball- abh P (2006) Astrocyte end-feet in germinal matrix, cerebral cortex, and white matter in developing infants. Pediatr Res 59:673-679.

Enge M, Bjarnegard M, Gerhardt H, Gustafsson E, Kalen M, Asker N, Hammes HP, Shani M, Fassler R, Betsholtz C (2002) Endotheliumspecific platelet-derived growth factor-B ablation mimics diabetic retinopathy. EMBO J 21:4307-4316.

Gee MS, Procopio WN, Makonnen S, Feldman MD, Yeilding NM, Lee WM (2003) Tumor vessel development and maturation impose limits on the effectiveness of anti-vascular therapy. Am J Pathol 162:183-193.

Hellstrom M, Gerhardt H, Kalen M, Li X, Eriksson U, Wolburg H, Betsholtz C (2001) Lack of pericytes leads to endothelial hyperplasia and abnormal vascular morphogenesis. J Cell Biol 153:543-553.

Heuchan AM, Evans N, Henderson Smart DJ, Simpson JM (2002) Perinatal risk factors for major intraventricular haemorrhage in the Australian and New Zealand Neonatal Network, 1995-97. Arch Dis Child Fetal Neonatal Ed 86:F86-F90.

Hirschi KK, D’Amore PA (1997) Control of angiogenesis by the pericyte: molecular mechanisms and significance. EXS 79:419-428.

Hungerford JE, Little CD (1999) Developmental biology of the vascular smooth muscle cell: building a multilayered vessel wall. J Vasc Res 36:2-27.

Investigators of the Vermont-Oxford Trial Network Database Project (1993) The Vermont-Oxford trials network: very low birth weight outcomes for 1990. Pediatrics 91:540-545.

Jain RK (2003) Molecular regulation of vessel maturation. Nat Med 9:685-693. 
Jain RK (2005) Antiangiogenic therapy for cancer: current and emerging concepts. Oncology (Williston Park) 19:7-16.

Kenny JD, Garcia-Prats JA, Hilliard JL, Corbet AJ, Rudolph AJ (1978) Hypercarbia at birth: a possible role in the pathogenesis of intraventricular hemorrhage. Pediatrics 62:465-467.

Kern TS, Engerman RL (1996) Capillary lesions develop in retina rather than cerebral cortex in diabetes and experimental galactosemia. Arch Ophthalmol 114:306-310.

Koga K, Todaka T, Morioka M, Hamada J, Kai Y, Yano S, Okamura A, Takakura N, Suda T, Ushio Y (2001) Expression of angiopoietin-2 in human glioma cells and its role for angiogenesis. Cancer Res 61:6248-6254.

Lindahl P, Johansson BR, Leveen P, Betsholtz C (1997) Pericyte loss and microaneurysm formation in PDGF-Bdeficient mice. Science 277:242-245.

Liu Y, Wada R, Yamashita T, Mi Y, Deng CX, Hobson JP, Rosenfeldt HM, Nava VE, Chae SS, Lee MJ, Liu CH, Hla T, Spiegel S, Proia RL (2000) Edg-1, the $G$ protein-coupled receptor for sphingosine-1-phosphate, is essential for vascular maturation. J Clin Invest 106:951-961.

Maxwell M, Galanopoulos T, NevilleGolden J, Hedley-Whyte ET, Antoniades HN (1998) Cellular localization of PDGF mRNAs in developing human forebrain. Neuropathol Appl Neurobiol 24:337-345.

Montuenga LM, Mariano JM, Prentice MA, Cuttitta F, Jakowlew SB (1998) Coordinate expression of transforming growth factor-betal and adrenomedullin in rodent embryogenesis. Endocrinology 139:3946-3957.

Ozerdem U (2006) Targeting pericytes diminishes neovascularization in orthotopic uveal melanoma in nerve/glial antigen 2 proteoglycan knockout mouse. Ophthalmic Res 38:251-254.

Sartore S, Chiavegato A, Faggin E, Franch R, Puato M, Ausoni S, Pauletto P (2001) Contribution of adventitial fibroblasts to neointima formation and vascular remodeling: from innocent bystander to active participant. Circ Res 89:1111-1121.

Shaheen RM, Tseng WW, Davis DW, Liu W, Reinmuth N, Vellagas R, Wieczorek AA, Ogura Y, McConkey DJ, Drazan KE, Bucana CD, McMahon G, Ellis LM (2001) Tyrosine kinase inhibition of multiple angiogenic growth factor receptors improves survival in mice bearing colon cancer liver metastases by inhibition of endothelial cell survival mechanisms. Cancer Res 61:1464-1468.

Shennan AH, Bewley S (2006) Why should preterm births be rising? BMJ 332:924-925.

Spiegel S, Milstien S (2003) Sphingosine-1-phosphate: an enigmatic signalling lipid. Nat Rev Mol Cell Biol 4:397-407.

Stoeltzing O, Ahmad SA, Liu W, McCarty MF, Wey JS, Parikh AA, Fan F, Reinmuth N, Kawaguchi M, Bucana CD, Ellis LM (2003) Angiopoietin-1 inhibits vascular permeability, angiogenesis, and growth of hepatic colon cancer tumors. Cancer Res 63:3370-3377.

Stratmann A, Risau W, Plate KH (1998) Cell type-specific expression of angiopoietin- 1 and angiopoietin-2 suggests a role in glioblastoma angiogenesis. Am J Pathol 153:1459-1466.

Tillet E, Vittet D, Feraud O, Moore R, Kemler R, Huber P (2005) $\mathrm{N}$-cadherin deficiency impairs pericyte recruitment, and not endothelial differentiation or sprouting, in embryonic stem cell-derived angiogenesis. Exp Cell Res 310:392-400.
Tomasek JJ, Gabbiani G, Hinz B, Chaponnier C, Brown RA (2002) Myofibroblasts and mechano-regulation of connective tissue remodelling. Nat Rev Mol Cell Biol 3:349-363.

Tommiska V, Heinonen K, Ikonen S, Kero P, Pokela ML, Renlund M, Virtanen M, Fellman V (2001) A national short-term follow-up study of extremely low birth weight infants born in Finland in 1996-1997. Pediatrics 107:E2.

Verbeek MM, de Waal RM, Schipper JJ, Van Nostrand WE (1997) Rapid degeneration of cultured human brain pericytes by amyloid beta protein. J Neurochem 68:1135-1141.

Volpe JJ (1989) Intraventricular hemorrhage in the premature infant-current concepts. Part II. Ann Neurol 25:109-116.

von Tell D, Armulik A, Betsholtz C (2006) Pericytes and vascular stability. Exp Cell Res 312:623-629.

Winkler F, Kozin SV, Tong RT, Chae SS, Booth MF, Garkavtsev I, Xu L, Hicklin DJ, Fukumura D, di Tomaso E, Munn LL, Jain RK (2004) Kinetics of vascular normalization by VEGFR2 blockade governs brain tumor response to radiation: role of oxygenation, angiopoietin-1, and matrix metalloproteinases. Cancer Cell 6:553-563.

Yamashita J, Itoh H, Hirashima M, Ogawa M, Nishikawa S, Yurugi T, Naito M, Nakao K (2000) Flk1-positive cells derived from embryonic stem cells serve as vascular progenitors. Nature 408:92-96.

Yancopoulos GD, Davis S, Gale NW, Rudge JS, Wiegand SJ, Holash J (2000) Vascular-specific growth factors and blood vessel formation. Nature 407:242-248.

Zhang L, Yang N, Park JW, Katsaros D, Fracchioli S, Cao G, O’Brien-Jenkins A, Randall TC, Rubin SC, Coukos G (2003) Tumor-derived vascular endothelial growth factor upregulates angiopoietin-2 in host endothelium and destabilizes host vasculature, supporting angiogenesis in ovarian cancer. Cancer Res 63:3403-3412. 